\title{
Morphology and band structure of orthorhombic PbS nanoplatelets: an indirect band gap material
}

\author{
David F. Macias-Pinilla,${ }^{\dagger, \ddagger}$ Carlos Echeverría-Arrondo, ${ }^{\ddagger}$ Andrés Fabián Gualdrón

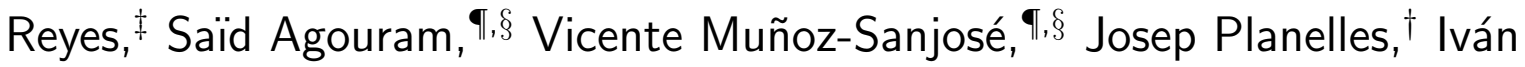

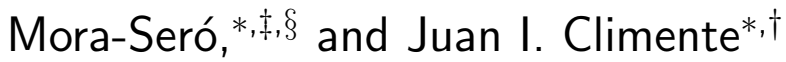 \\ $\dagger$ Departament de Química Fisica i Analítica, Universitat Jaume I, Av. Sos Baynat, s/n, \\ 12071 Castelló, Spain \\ $\ddagger$ Institute of Advanced Materials (INAM), Universitat Jaume I, Av. Sos Baynat, s/n, \\ 12071 Castelló, Spain \\ 9Department of Applied Physics and Electromagnetism, University of Valencia, 46100 \\ Valencia, Spain \\ $\S$ Materials for Renewable Energy (MAER), Unitat Mixa d'Investigació UV-UJI \\ E-mail: sero@uji.es; climente@uji.es
}

\begin{abstract}
$\mathrm{PbS}$ quantum dots and nanoplatelets (NPLs) are of enormous interest for the development of optoelectronic devices. However, some important aspects of their nature remain unclear. Recent studies have revealed that colloidal PbS NPLs may depart from the rock-salt crystal structure of bulk, and form an orthorhombic (Pnma) modification instead. To gain insight into the implications of such a change over the optoelectronic properties, we have synthesized orthorhombic PbS NPLs and determined the lattice parameters by means of selected area electron diffraction measurements. We have then calculated the associated band structure using density functional theory
\end{abstract}


with Perdew-Burke-Ernzerhof functional for solids and with the GW approximation, including spin-orbit interaction. An indirect band gap is found, which may explain the weak luminescence reported in experiments. We derive effective masses for conduction and valence bands, and deduce that quantum confinement along the $a$ crystallographic axis (short axis of the NPL) reinforces the indirect band gap, but that along $b$ and $c$ axes favors a direct gap instead. Calculations for colloidal nanoplatelets of $1.8 \mathrm{~nm}$ thickness, carried out with k·p theory, show that excitonic effects are strong, with binding energies of about $150 \mathrm{meV}$.

\section{Introduction}

$\mathrm{PbS}$ quantum dots have attracted much interest due to the possibility of tuning their bandgap in the NIR region through control of the size, ${ }^{1}$ thus extending light absorption beyond the visible spectrum. Consequently, $\mathrm{PbS}$ dots have been extensively employed in the development of optoelectronic devices as solar cells, ${ }^{2}$ LEDs $^{3}$ or photodetectors. ${ }^{4,5}$ Recently, the synergistic combination with halide perovskites has been also explored, showing influence of $\mathrm{PbS}$ dots on the morphology of the halide perovskite, producing an increase of solar cell performance ${ }^{6,7}$ and a significant increase on the long term device stability, ${ }^{8}$ as well as advanced LEDs ${ }^{9}$ and photodetectors. ${ }^{10,11}$

The use of NPLs instead of dots offers additional possibilities for optoelectronic applications, which follow from their quasi-two-dimensional structure. These include large exciton binding energies, which are convenient for room temperature devices, giant oscillator strength enabling extremely bright emission, and extremely narrow emission linewidth, resulting from the precise control of the NPL thickness. ${ }^{12-14}$ Specifically, PbS NPLs have been shown to combine a relatively narrow band gap, which gives access to near infrared photodetection and emission, ${ }^{15-17}$ with other attractive properties, such as low-threshold carrier multiplication, ${ }^{18}$ gate-controlled spin-orbit interaction, ${ }^{19}$ strong multiquantum-well coupling, ${ }^{20,21}$ stable dielectric response, ${ }^{22}$ high surface homogeneity and efficient cross-linking, ${ }^{23}$ and facile 
realization of topological and valleytronic effects. ${ }^{24}$ Very recently, we have also shown that the addition of PbS NPLs increases the long term stability of unencapsulated perovskite solar cells as well as the performance and reproducibility of perovskite solar cells prepared at ambient conditions. ${ }^{25}$ Despite the high interest in these systems, there are some aspects of PbS NPLs that remain unclear.

While early works on PbS NPLs assumed or inferred rock-salt crystal structure (as in bulk and nanocrystals), later experimental studies have evidenced that orthorhombic (Pnma space group) modification may be also formed. ${ }^{15,16}$ Orthorhombic NPLs seem to present a distinct optical response, namely weak luminescence at $650-800 \mathrm{~nm},{ }^{15,16}$ which is much shorter than the emission wavelength often reported for rock-salt NPLs $(1200-1500 \mathrm{~nm})$ despite having similar thickness. Short wavelength emission was also observed in earlier studies of PbS NPLs, whose crystal structure was not unambiguously defined. ${ }^{17,20}$ The nature of such a different optical response is still an open question. Previous theoretical studies about the electronic properties of PbS NPLs have mostly focused on the cubic modification, using either density function theory (DFT) $)^{19,23,24,26}$ or k.p models. ${ }^{27}$ Recently, Akkerman and co-workers calculated the band structure of orthorhombic PbS NPLs, within DFT schemes. ${ }^{16}$ Their results suggest that the band structure is very similar to that of rock-salt NPLs, both showing a direct gap of similar energy. This result is in contrast with that reported for SnS and GeS NPLs, which crystallize in the same type of lattice, but exhibit indirect bandgaps. ${ }^{28}$ It also leaves the seemingly distinct optical features of the orthorhombic PbS NPLs unexplained.

The band structure of bulk PbS in Pnma phase has been calculated using DFT by Zagorac et al. ${ }^{29}$ A direct band edge gap of $0.7 \mathrm{eV}$, wider than that in rock-salt $\mathrm{PbS}(0.4 \mathrm{eV})$, was obtained. While the increase in band gap $\left(\Delta E_{g}=0.3 \mathrm{eV}\right)$ is qualitatively consistent with the shorter wavelength of orthorhombic NPLs, it is much smaller than the empirical value, $\Delta E_{g} \approx 0.8 \mathrm{eV}$. Obviously, for comparison with actual NPLs, the role of quantum confinement must be addressed, as it is a major energetic contribution to the optical gap. ${ }^{26,30,31}$ Besides, 
the calculations missed spin-orbit interactions, which are certainly important in lead, and were performed in the local density approximation (LDA), which is particularly unsuited to estimate band gaps. ${ }^{32}$

To gain further insight into the photophysics of orthorhombic PbS NPLs, in this work we synthesize PbS NPLs, analyze the crystallographic structure, and use DFT and k·p modelling to analyze the associated electronic structure and optical transitions. From transmission electron microscopy (TEM) and selected area electron diffraction (SAED) measurements we determine the crystallographic phase and unit cell parameters. From DFT, we calculate the associated bulk band structure -including spin-orbit interaction-, and obtain effective masses in the valleys of interest. These masses are then plugged into effective mass Hamiltonians to account for quantum confinement and to quantify excitonic properties.

We find that the bulk band structure shows a band gap of $0.5 \mathrm{eV}$, similar to that of rocksalt $\mathrm{PbS}$, but of indirect nature. From the effective masses, we deduce that the indirect band edge character is reinforced by quantum confinement along the $a$ axis of the lattice (short axis $(x)$ of the NPL), but it can switch to direct if strong confinement takes place in orthogonal directions. The exciton binding energy and Bohr radius are found to be comparable to those in cubic NPLs, which translates into weak influence of lateral confinement beyond $\sim 15 \mathrm{~nm}$ in-plane sides (longitudinal $(y)$ and lateral $(z)$ dimensions). The potential connection of the above results with spectroscopic experiments is discussed.

\section{Experimental Section}

\section{Synthesis of PbS Nanoplatelets}

PbS NPLs were prepared according to an established procedure. ${ }^{15}$ Briefly, potassium hydroxide and octadecanol were mixed in a 1:1 molar ratio at $150{ }^{\circ} \mathrm{C}$ under air. After cooling down to room temperature, carbon sulfide was injected swiftly. In this way, potassium octadecylx-

anthate (KOctdX) is formed. Then, lead octadecylxanthate (PbOctdX) was synthesized by 
mixing an aqueous solution of lead nitrate and KOctdX in an equimolar amount under vigorous stirring. The final product was filtered and washed with Milli-Q water for several times. For the NPLs synthesis, $90 \mathrm{mg}$ PbOctdX and $5 \mathrm{~mL}$ trioctylamine (TOA) were mixed in a 50 $\mathrm{mL}$ three-neck flask for $30 \mathrm{~min}$ at room temperature under vacuum. Then, the temperature of the reaction mixture was slowly increased to $80{ }^{\circ} \mathrm{C}$ under a $\mathrm{N}_{2}$ atmosphere. It took around 15 min to reach the desired temperature. With the purpose to vary the dimensions of the NPLs, the reaction was conducted for $0.5,1,3$ and $5 \mathrm{~h}$, observing a change from orange to a darker color. After the NPLs synthesis was completed, the reaction was cooled down to room temperature, and $2 \mathrm{~mL}$ of toluene, $1 \mathrm{~mL}$ of oleic acid and $3 \mathrm{~mL}$ of 1,2-dichlorobenzene were added. The NPLs were next purified by adding excess of acetonitrile and centrifuged at $5000 \mathrm{rpm}$ for $5 \mathrm{~min}$. The NPLs were then dispersed in toluene and again precipitated by adding acetonitrile and centrifuged at $5000 \mathrm{rpm}$ for $5 \mathrm{~min}$. Finally, the purified NPLs were dispersed in hexane.

\section{Morphological and optical characterization}

TEM images and SAED patterns of PbS NPLs were achieved by a field emission gun TECNAI G2 F20 microscope operated at $200 \mathrm{kV}$. Average dimensions of the NPLs were obtained from the TEM images with ImageJ software. UV-Vis absorption spectra of NPLs solutions at different reaction times were acquired by using a PerkinElmer UV/VIS/NIR spectrometer Lambda $1050+$. The wavelength range for the measurements was 450-1050 nm. Steady state photoluminescence (PL) measurements were conducted through a photoluminescence spectrophotometer (Fluorolog 3-11, Horiba). An excitation wavelength of $420 \mathrm{~nm}$ was used to perform the steady state PL in a wavelength range between $600-800 \mathrm{~nm}$. For the calculation of the cell parameters from SAED patterns, we used the known Miller indixes $(h k l)$ and the equation for the interplanar distance $d$ in orthorhombic structures:

$$
\frac{1}{d^{2}}=\frac{h^{2}}{a^{2}}+\frac{k^{2}}{b^{2}}+\frac{l^{2}}{c^{2}}
$$


with $a, b, c$ the cell parameters to calculate.

\section{Theoretical Methods}

The band gap of rock-salt NPLs has been well described within DFT using a Perdew-BurkeErnzerhof (PBE) exchange-correlation functional, with full inclusion of spin-orbit coupling (SOC). ${ }^{26}$ To study the Pnma modification, which in bulk form is stable at high pressure, ${ }^{29,33}$ we used a PBEsol functional instead, which is known to be more accurate for solids under pressure, ${ }^{34}$ also with SOC inclusion. By using the generalized gradient approximation (GGA), both PBE and PBEsol outperform bare LDA in estimating bulk band gaps. ${ }^{35}$ Where necessary, to confirm the robustness of the results, the calculations at the PBEsol level were compared against PBE functional and GW approach.

Rather than calculating the band structure of few-monolayer NPLs, we calculated that of bulk PbS. In this way, we exploited the symmetry of the lattice to carry out numerically accurate estimates. Quantum confinement was introduced in a second stage using singleband (effective mass) k.p theory, which has proved successful in quantitatively describing the photophysics of thin NPLs prepared out of other materials. ${ }^{27,30,31,36}$ This approach also circumvents possible problems of atomistic models related to uncertainties on the hybrid (inorganic NPL-organic ligand) surface details, for which experimental information is limited.

DFT calculations were performed with the Quantum Espresso code. ${ }^{37}$ We geometrically relaxed the experimental PbS othorhombic unit cell (ICSD 648451) until forces on the individual nuclei were less than $0.001 \mathrm{R}_{\mathrm{y}} / \mathrm{a}_{0}$, where $\mathrm{R}_{\mathrm{y}}$ is the Rydberg constant and $\mathrm{a}_{0}$ the Bohr radius. The first Brillouin zone was sampled with a $\Gamma$-centered Monkhorst-pack grid of $4 \times 8 \times 8 \mathrm{k}$ points. In order to avoid possible phase transitions, we controlled cell dynamics in the relaxation process with the Wentzcovitch Extended Lagrangian. ${ }^{38}$ We also run the Yambo code, ${ }^{39,40}$ which implements the GW approximation. ${ }^{41,42}$

To study optoelectronic properties we described excitons with effective mass Hamilto- 
nians, including dielectric mismatch between the NPL and the surrounding ligands -which is very important in colloidal platelets ${ }^{21,43-45}$-, lateral confinement -which can be sizable in PbS NPLs ${ }^{15}$ - and electron-hole Coulomb interaction via a variational method, which provides reliable description of correlations. Further details of this model can be found in Refs. 43 and 46.

\section{Results}

\section{PbS Orthorhombic Structure}

Our first goal was to confirm the orthorhombic phase of PbS NPLs. We synthesized NPLs following Khan and co-workers. ${ }^{15}$ Similar to Ref. 15, with increasing reaction time we see one direction with fixed dimensions and two that increase gradually, see Fig. 1a-d (histograms of these dimensions are provided in Fig. S4 of the Supporting Information (SI)). Following the HRTEM analysis of Ref. 15, the fixed direction is assigned to the (100) facet and the other two to the (001) and (010) facets. Consequently, we conclude that $a-, b$ - and $c$-crystallographic directions correspond to the thickness, length and width of PbS NPLs, respectively. Through SAED patterns, interplanar spacings $(d)$ associated to characteristic diffraction planes were calculated in order to determinate the experimental cell parameters of PbS NPLs. According to the $d$ values estimated from SAED rings (insets of Fig. 1), the corresponding diffraction planes found for the synthesized PbS NPLs are $(200) /(020) /(002)$ from the cubic structure (ICSD 38293 card), while the (400), (002) and (020) planes are ascribed to the extended (100) (001) (010) facets in the orthorhombic phase (ICSD 648451 card). By using the relationship between the interplanar distance for orthorhombic structures described in Equation 1, we determined the corresponding $a$ - $b$ - and $c$-parameters from the above mentioned diffraction planes.

The presence of a cubic phase is attributed to the formation of some $\mathrm{PbS}$ nanoparticles together with NPLs. Nevertheless, since the capping ligands used during the synthesis promote 
the laminar growth of NPLs from the attachment of monomers or small PbS nanoparticles, ${ }^{47}$ the orthorhombic phase is the main crystalline structure of the materials. This fact can be confirmed through UV-Vis absorption and photoluminescence (PL) measurements obtained from NPLs samples (see Fig. S5). An absorption shoulder at $720 \mathrm{~nm}$ was distinguished, and then red-shifted to $736 \mathrm{~nm}$ by increasing the time of NPLs synthesis. Furthermore, the typical PL peak of 2D PbS NPLs was reached between 736-741 nm, similar to previous works. $^{15}$

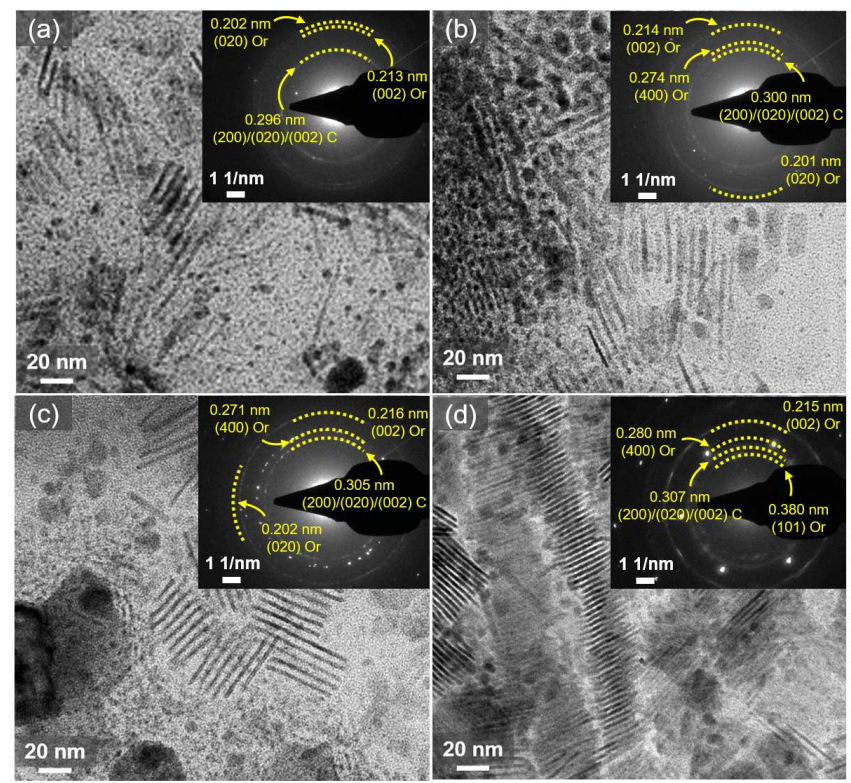

Figure 1: Typical TEM images of the PbS NPLs synthesized at (a) 0.5 h, (b) 1.0 h, (c) $3.0 \mathrm{~h}$ and (d) $5.0 \mathrm{~h}$. SAED patterns of the corresponding PbS NPLs depicted in the Insets of Fig. 1 show the diffraction planes of cubic phase (ICSD 38293) and orthorhombic phase (ICSD 648451) of the PbS NPLs.

We next attempted to model the same orthorhombic lattice (ICSD 648451) using DFT. The first attempts to relax the crystal structures inevitably led to a phase change from orthorhombic to rock-salt. This fact reflects that rock-salt is more stable and that the orthorhombic phase is achieved by external factors in the environment. Previous studies preserved the orthorhombic phase by keeping the cell parameters fixed. ${ }^{16}$ We opted to carry out the relaxation process using the Wentzcovitch Extended Lagrangian instead, which is useful for the cell parameters optimization and improves numerical simulations within a single 
phase, allowing a slight freedom on the cell parameters. ${ }^{38}$ The cell parameters obtained using PBE and PBEsol functionals approximately match the experimental ones, and are listed in Table 1.

Table 1: Cell parameters of orthorhombic PbS NPLs measured with SAED at different times of reaction during the synthesis, and those of bulk $\mathrm{PbS}$ as obtained from the ICSD data base, and from calculations run with PBE and PBEsol exchange-correlation functionals.

\begin{tabular}{cccc} 
Method / reaction time & $\mathrm{a}(\AA)$ & $\mathrm{b}(\AA)$ & $\mathrm{c}(\AA)$ \\
\hline Exp. / 0.5 h & - & 4.04 & 4.26 \\
Exp. / 1.0 h & 10.96 & 4.02 & 4.28 \\
Exp. / 3.0 h & 10.84 & 4.04 & 4.32 \\
Exp. / 5.0 h & 11.20 & - & 4.30 \\
ICSD 648451 & 11.28 & 4.02 & 4.29 \\
PBE & 11.31 & 4.03 & 4.29 \\
PBEsol & 11.31 & 4.04 & 4.29
\end{tabular}


(a) ICSD 648451
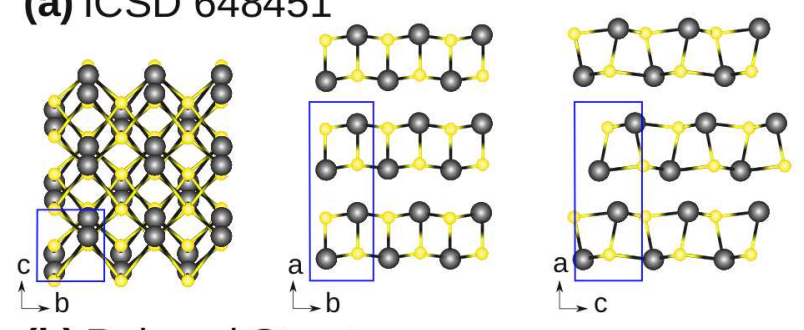

(b) Relaxed Structure
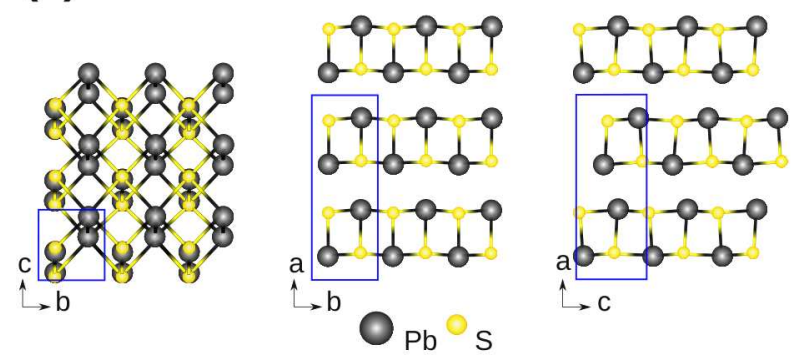

(c) XRD

(d) Brillouin Zone
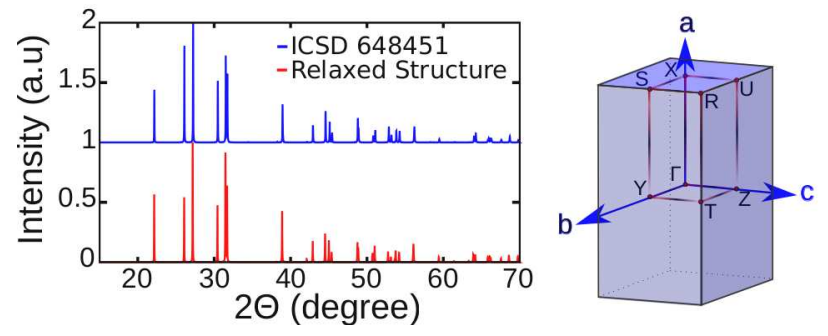

Figure 2: (a) Experimental unit cell (ICSD 648451), (b) Geometrically relaxed unit cell (PBEsol). (c) XRD of experimental (blue) and calculated (red) bulk orthorhombic PbS. (d) First Brillouin zone of a crystal with orthorhombic symmetry.

Fig. 2a-b evidences the similarity between the experimental crystal structure (ICSD 648451) and that relaxed with the PBEsol functional. Comparing the X-ray diffraction (XRD) patterns (Fig. 2c) obtained by VESTA, ${ }^{48}$ the matching between the two structures is clear. The orthorhombic features of the XRD pattern have also been reported in previous experiments on PbS NPLs. ${ }^{15,16}$

\section{Band Structure}

We started by calculating the energy bands of bulk (Pnma) PbS with PBEsol functional following the paths between high symmetry points in the first Brillouin zone, that we depict in Fig. 2d. As usual in DFT, the absolute values of the band gaps may be offset from 
experimental ones, but the predicted trends are usually reliable. ${ }^{23,24,26}$ In Fig. 3 we compare the energy bands with and without SOC. The figure shows that SOC, which was missing in some earlier works, ${ }^{29}$ has a non-negligible influence due to the presence of a heavy metal, lead in this case. It gives rise to a sizable narrowing of the band gap between valence and conduction bands. Similar results were obtained with PBE functional (See Fig. S2). Hereafter, all the reported calculations include SOC.

(a) PbS bands

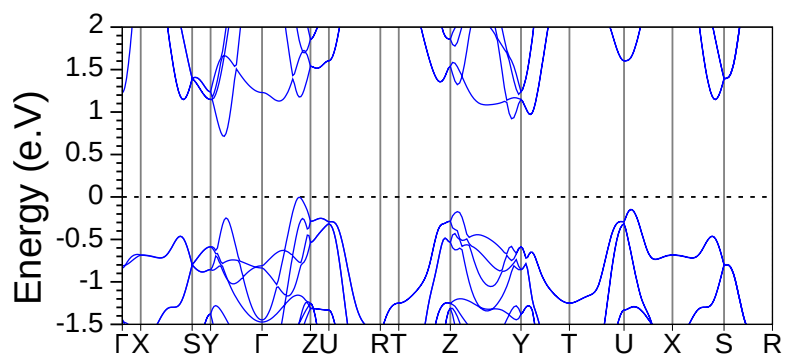

(b) PbS bands + SOC

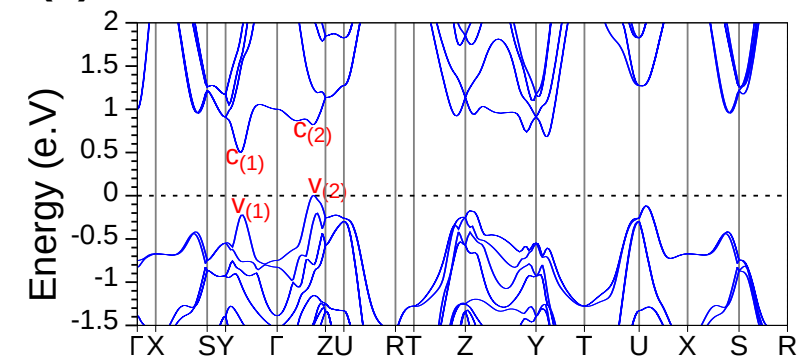

Figure 3: Electronic band spectra of bulk orthorhombic PbS calculated at the PBEsol level (b) with and (a) without SOC. Note the expected narrowing of the band gaps when including SOC.

The most relevant feature in Fig. 3 is related to the relative gap values for different valleys: the fundamental band gap is found to be indirect. The conduction band minimum is found near the $Y$ point (labeled as $c_{(1)}$ in Fig. $3 \mathrm{~b}$ ) and the valence band maximum near the $Z$ point instead (labeled as $v_{(2)}$ in Fig. 3b). The indirect band gap is consistent with that reported in most orthorhombic structures, ${ }^{28,49-51}$ but it is in contrast to the direct gap predicted in Ref. 29 for bulk PbS and in Ref. 16 for a four-monolayer PbS NPL. The functional used in Ref. 29 was LDA, however, that of Ref. 16 was HSE06, -a hybrid functional including a portion of the exact Hartree-Fock exchange-correlation functional-, which is much more 
computationally expensive and accurate. To confirm the robustness of our results, we further calculate the band gap of bulk orthorhombic $\mathrm{PbS}$ with the GW approximation, which implements quasiparticle self-energy corrections in the framework of many-body perturbation theory. This method is known to outperform generalized gradient approximations in determining lead chalcogenides band gaps, ${ }^{29,52}$ and has been shown to agree quantitatively with experimental values of bulk in rock-salt PbS. ${ }^{23}$ Because of the high computational cost of such calculations, we restrict to the energy bands within the the path $Y-\Gamma-Z$ of the Brillouin zone. The resulting energy bands (Fig. 4) agree with those of PBEsol and PBE, thus providing additional support for the indirect character of the band gap. Table 2 summarizes the gap energies between different band edges found with each method.

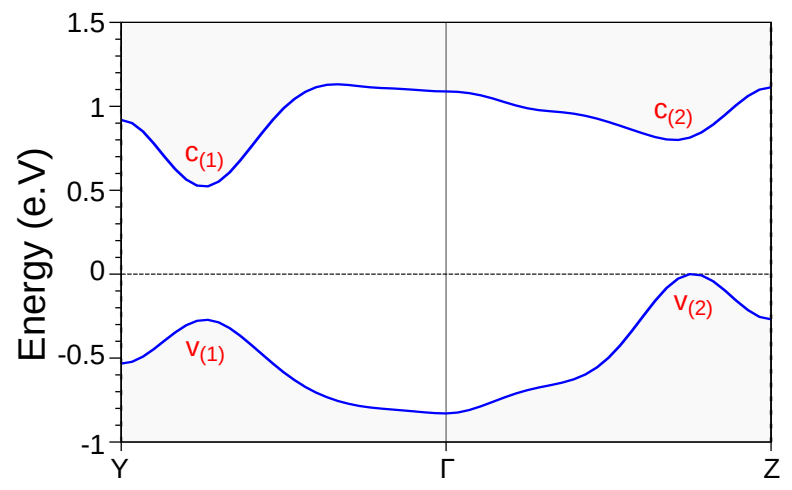

Figure 4: Bulk energy bands from GW+SOC approximation.

Table 2: Energy gaps obtained from PBEsol+SOC, PBE+SOC and GW+SOC.

\begin{tabular}{cccc} 
BANDS & \multicolumn{3}{c}{ Band gap (eV) } \\
& PBEsol & PBE & GW \\
\hline $\mathrm{c}_{(1)}-\mathrm{v}_{(1)}$ & 0.72 & 0.76 & 0.78 \\
$\mathrm{c}_{(1)}-\mathrm{v}_{(2)}$ & 0.5 & 0.54 & 0.52 \\
$\mathrm{c}_{(2)}-\mathrm{v}_{(1)}$ & 1.14 & 1.06 & 1.08 \\
$\mathrm{c}_{(2)}-\mathrm{v}_{(2)}$ & 0.82 & 0.83 & 0.8
\end{tabular}

On the other hand, the origin of the direct band gap for orthorhombic PbS NPLs in the calculations of Ref. 16 traces back to the choice of quasi-tetragonal cell parameters for PbS ( $a=11.90 \AA, c=4.22 \AA, b=4.20 \AA$ ). We have tested that our (bulk) calculations retrieve direct band gap behavior in this limit as well (see Fig. S6). The choice of such cell parameters 
in their work was driven by the best fit to powder XRD patterns. By contrast, the SAED measurements of our NPLs reveal a more pronounced orthorhombic lattice ( $a=11.20 \AA$, $b=4.02 \AA, c=4.29 \AA$ ). Interestingly, this small increase in lattice anisotropy suffices to switch the calculated bulk band gap from direct to indirect, the latter being $\sim 0.22 \mathrm{eV}$ smaller than the former.

The different lattice constants in our NPLs as compared to those of Ref. 16 might be related to differences in the measurement technique or the synthetic route itself. The different synthetic routes produce not only structures with very different lateral dimensions (NPLs with $49 \mathrm{~nm} \times 2.5 \mathrm{~nm}$ sides in our case, nanosheets with $1.4 \mu \mathrm{m} \times 0.3 \mu \mathrm{m}$ sides in Ref. 16), but also the emergence of both orthorhombic NPLs and rock-salt PbS nanocrystals, which may hamper the determination of lattice constants. In this regard, SAED offers the advantage of selecting nanoparticles of interest with well-defined orientation, determined with TEM analysis. It is worth noting that the resulting lattice parameters we obtain are closer to those of ICSD database for bulk orthorhombic PbS.

Figure 5 shows an isosurface of constant charge density for the band edge states of interest. From these spatial distributions of charge densities we conclude that the valence band edge orbitals have $\left|P_{y}\right\rangle$ and $\left|P_{z}\right\rangle$ character for $v_{(1)}$ and $v_{(2)}$, respectively, and are mostly localized at the place of S atoms. Conduction band orbitals have more $|S\rangle$-like character, and are shared by $\mathrm{Pb}$ and $\mathrm{S}$ atoms. Because valence and conduction band orbitals differ in one quantum of angular momentum, Fig. 5 implies that, from the unit cell (periodic Bloch function) symmetry, all different direct and indirect transitions are in principle allowed with due polarization of light. If translational symmetry holds, however, indirect transitions will be forbidden by conservation of in-plane $(y z)$ linear momentum. In PbS NPLs, lateral confinement lifts the translational symmetry but, as we shall show below, sizable confinement is only obtained for lateral dimensions under 10-15 nm. For NPLs above these dimensions, indirect transition should remain severely forbidden. For smaller NPLs, coupling between non-degenerate valleys may relax the selection rule. However, tight-binding studies on strongly confined 
PbS nanocrystals suggest that valley coupling is weak for band-edge states. ${ }^{53,54}$ The indirect transition is then likely to stay largely quenched.

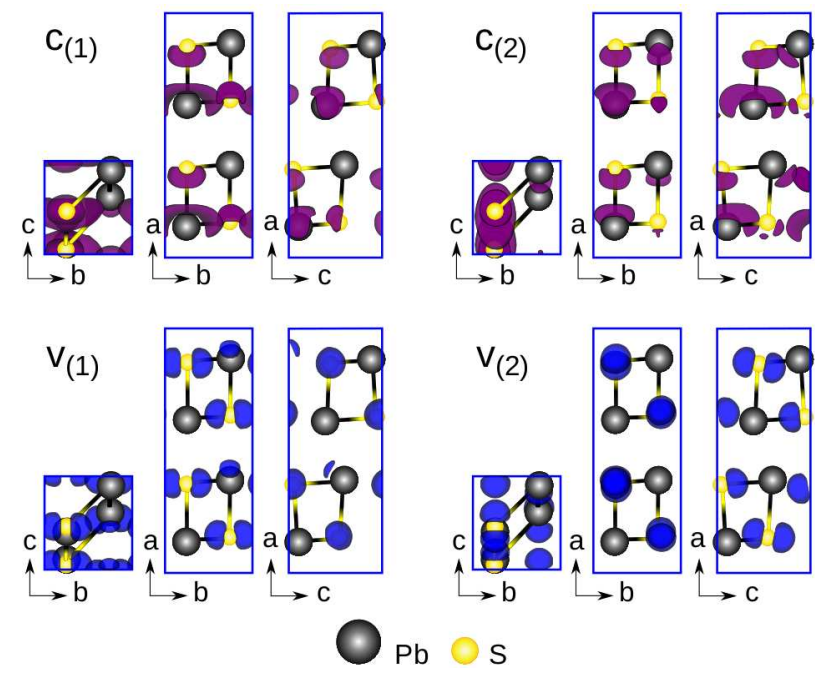

Figure 5: Isosurfaces of constant charge density in the band-edge states of interest, obtained with PBEsol functional, where blue stands for valence and magenta stands for conduction.

Effective masses have proved useful to estimate several material properties of practical interest with simple (continuum) models. ${ }^{55-58}$ We thus extracted effective masses for orthorhombic PbS from our atomistic calculations. Assuming parabolic energy dispersion around the band edge, the energy at a discrete wave vector point $\mathbf{k}_{s}$ can be expressed as:

$$
E\left(\mathbf{k}_{\mathbf{s}}\right)=\frac{\hbar^{2} k_{s}^{2}}{2 m^{*}}
$$

where $m^{*}$ is the effective mass. Diagonal values of the mass, $m_{i}^{*}$ were obtained by taking five points $E\left(\mathbf{k}_{\mathbf{s}}\right)$ along the $k_{i}$ axis (separated from each other by $200 /(a, b, c) \AA^{-1}$ ), centered around the band edge wave vector, and fitting the effective mass value. We checked that quantitatively similar results were obtained by fitting the second derivative of the band edge with finite difference derivations. Table 3 summarizes the effective masses obtained for the valleys of interest along different crystallographic directions. Similar effective masses are found with the PBE functional as given in Table S1 (SI). As in the case of rock-salt structures, ${ }^{59}$ masses are highly anisotropic, with masses in one direction ( $x$, in our case) 
being very different from the other two. The two latter present small differences, indicating that the reduced symmetry in the $y, z$ directions has only moderate impact on the masses.

Table 3: Effective Masses obtained from energy bands with PBEsol+SOC, where $m_{0}$ is the free electron mass.

\begin{tabular}{|c|c|c|c|}
\hline \multirow{2}{*}{ BAND } & \multicolumn{3}{|c|}{ Effective Mass $\left(\mathrm{m}_{0}\right)$} \\
\hline & $\mathbf{m}_{\mathrm{x}}^{*}$ & $\mathbf{m}_{\mathrm{y}}^{*}$ & $\mathbf{m}_{\mathbf{z}}^{*}$ \\
\hline$c_{(1)}$ & 0.329 & 0.148 & 0.153 \\
\hline$c_{(2)}$ & 0.275 & 0.266 & 0.312 \\
\hline $\mathrm{V}_{(1)}$ & 0.338 & 0.158 & 0.183 \\
\hline $\mathrm{v}_{(2)}$ & 1.423 & 0.254 & 0.215 \\
\hline
\end{tabular}

Quantum confinement plays an important role in determining emission energies. Its effects can be qualitatively anticipated from the masses in Table 3. For example, the heavier masses of $c_{(1)}$ and $v_{(2)}$ (HOMO and LUMO in the system) along the crystallographic axis $a\left(m_{x}\right)$, as compared to the other bands, imply that confining the NPL in that crystallographic direction reinforce the indirect character of the band edge transition. By contrast, confinement along the in-plane $b$ or $c$ axis would favors direct transitions instead.

\section{Emission Energy and dielectric effects}

With the aim of studying the optical properties of orthorhombic PbS NPL, we build on the previous results of bulk, but now adding quantum confinement and electron-hole (exciton) Coulomb interaction. In this section we study the influence of such effects. 

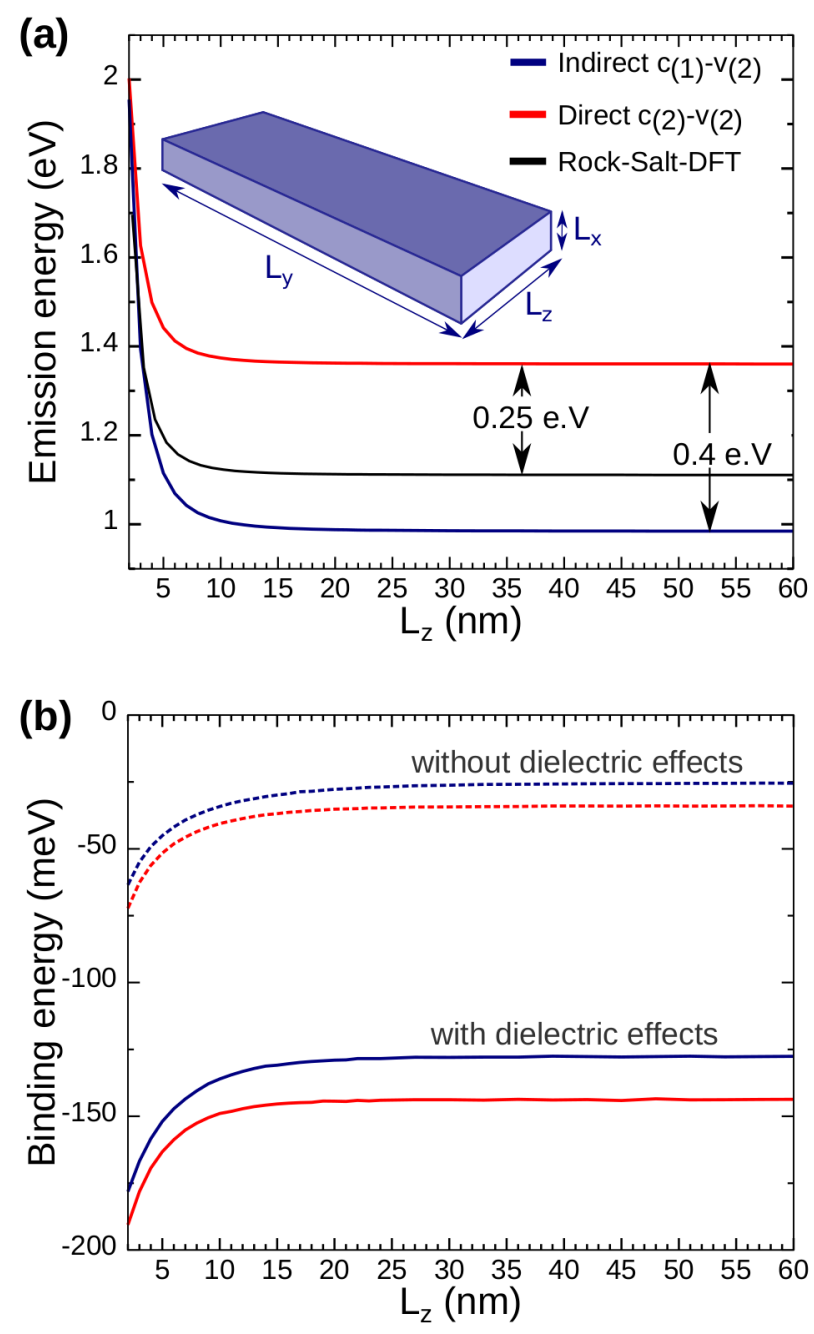

Figure 6: (a) Exciton emission energies vs lateral confinement ( $z$ direction) in orthorhombic PbS NPLs with direct (red) and indirect gap (blue), and that in rock-salt NPL (black). A crossover occurs when the lateral confinement is comparable to the vertical confinement $(x$ direction). (b) Binding energies for direct (red) and indirect (blue) exciton with (solid lines) and without (dashed lines) dielectric mismatch effects vs lateral confinement. Notice the strong influence of dielectric confinement. All calculations correspond to NPLs with thickness $L_{x}=1.8 \mathrm{~nm}$ and $L_{y}=50 \mathrm{~nm}$. Gaps and masses were computed with the PBEsol functional; exciton eigenenergies were obtained within $\mathrm{k} \cdot \mathrm{p}$ theory.

Using a four-band k·p Dimmock Hamiltonian for rock-salt structures ${ }^{8,59}$ we checked that conduction-valence band coupling is small in NPLs despite the narrow band gaps of bulk $\mathrm{PbS}$, because quantum confinement introduces opens the gap up to values exceeding $1 \mathrm{eV}$. Since bulk band gaps and effective masses are of the same order, it is reasonable to expect the same behavior in orthorhombic NPLs. Thus, we safely model the system using single 
band Hamiltonians ${ }^{43}$ built up with the calculated gaps of Table 2 and the effective masses of Table 3 (following Refs. 15,16, $x$ is the direction of strong confinement). Dielectric constants $\epsilon_{1}=17.2$ and $\epsilon_{2}=2.5$ were used for $\mathrm{PbS}^{60}$ and ligands ${ }^{61,62}$ respectively.

In Fig. $6 \mathrm{a}$ and $\mathrm{b}$ we analyze the effect of lateral confinement (in $z$ axis) on the exciton emission energy and binding energy, respectively, for a NPL with $L_{x}=1.8 \mathrm{~nm}$, corresponding to $\sim 7 \mathrm{PbS}$ monolayers, which is the thickness of the NPLs synthesized in Ref. 15. The length in $L_{y}$ is fixed at $50 \mathrm{~nm}$, well above the $2 \mathrm{D}$ exciton Bohr radius $\left(a_{B}^{*} \approx 3.6 \mathrm{~nm}\right.$, vide infra) so that lateral confinement is introduced exclusively by the variable $L_{z}$.

The blue line in Fig. 6a shows the band edge (indirect, $\left.c_{(1)}-v_{(2)}\right)$ transition energy. In the quantum well limit $\left(L_{z}>15 \mathrm{~nm}\right)$, vertical confinement pushes the emission energy to $\sim 1 \mathrm{eV}$, i.e. about twice larger than in bulk. This is in spite of the large binding energy $(\sim 0.125 \mathrm{eV}$ if dielectric mismatch is taken into account, blue solid line in Fig. 6b). Sizable influence of lateral confinement is only observed for $L_{z}<15 \mathrm{~nm}$, when emission energy starts blueshifting and exciton binding energies are enhanced.

For comparison, in Fig.6a we also plot the direct $c_{(2)}-v_{(2)}$ transition (red line). For large area NPLs, the direct band gap exceeds the indirect one by $\sim 0.4 \mathrm{eV}$. This splitting is about twice larger than the $0.22 \mathrm{eV}$ observed for bulk in Table 3 and confirms that quantum confinement along the $a$ axis reinforces the indirect nature of the band. Yet, $c_{(2)}$ electrons have heavier in-plane masses than $c_{(1)}$ ones (recall Table 3). One may thus expect that for strong lateral confinement the ground state switches from indirect to direct. Figure 6a shows that this can actually happen, but only for very strong lateral confinement $\left(L_{z}<3 \mathrm{~nm}\right)$. The black line in Fig.6a corresponds to rock-salt NPLs (calculated bulk band gap and masses can be found in Sec.III of SI). Clearly, the cubic lattice is an intermediate case between the direct and indirect gaps of orthorhombic PbS NPLs.

Figure $6 \mathrm{~b}$ shows that binding energies in orthorhombic NPLs with $L_{z}>15 \mathrm{~nm}$ are similar to those estimated by Yang and Wise for thin 2D rock-salt nanosheets, ${ }^{27}$ and also display a strong enhancement arising from dielectric mismatch. With regard to total exciton energy, 
we find such an enhancement is actually exceeded by the increase of self-energy (not shown), which leads to a moderate blueshift of the emission energy, similar to that reported for cubic PbS NPLs. ${ }^{27}$ The binding energy of direct excitons is found to be slightly larger than that of indirect ones, because the electron and hole wave functions are more similar (cf. in-plane masses in Table 3).

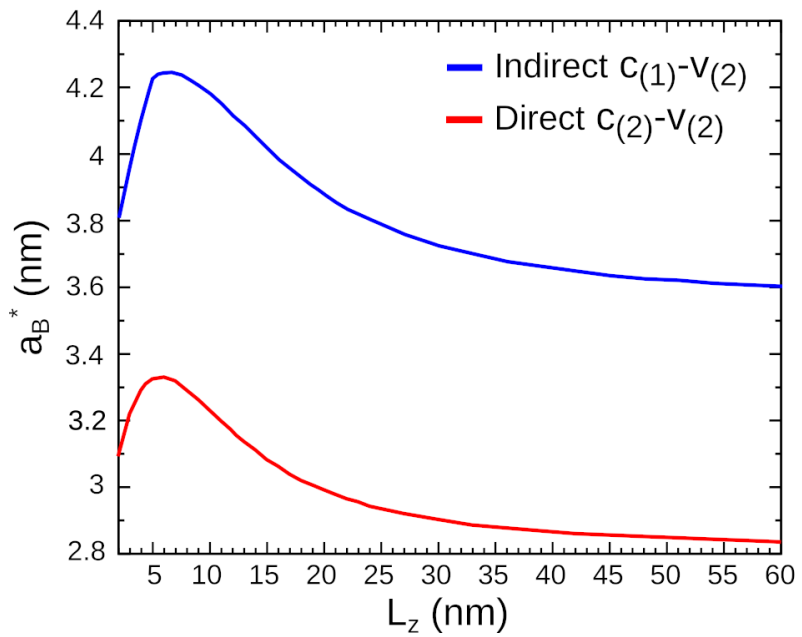

Figure 7: Effective exciton Bohr radius in NPLs vs lateral confinement. Indirect gap (ground state) and direct gap excitons are compared.

From our variational calculation of the exciton energy, we can also extract the effective Bohr radius, $a_{B}^{*}{ }^{43}$ Fig. 7 shows the corresponding values as a function of the NPL lateral size. For weak lateral confinement, the $2 \mathrm{D}$ limit is retrieved, which amounts to $a_{B}^{2 D}=3.6 \mathrm{~nm}$ for indirect excitons and to $a_{B}^{2 D}=2.82 \mathrm{~nm}$ for direct ones. The smaller radius for the direct exciton reflects the larger binding energy. When $L_{z}$ decreases, $a_{B}^{*}$ first becomes larger, owing to the enhanced binding energy (Fig. 6b), but then decreases due to quantum confinement. This behavior is expected from the approximate scaling as $1 / L_{z}$ and $1 / L_{z}^{2}$ for Coulomb and quantum confinement energies.

The effect of NPL thickness on the band edge is illustrated in Fig. 8, for a platelet with negligible lateral confinement $\left(L_{y}=L_{z}=50 \mathrm{~nm}\right)$. In thin structures, deviations of the effective mass from bulk parabolic values make single-band $\mathrm{k} \cdot \mathrm{p}$ models overestimate quantum confinement effects, ${ }^{44}$ but these are still able to provide semi-quantitative trends. ${ }^{27,31}$ The 
figure shows that both direct (red line) and indirect (blue line) gaps decrease rapidly with the number of orthorhombic PbS monolayers. The effect is however more pronounced for cubic PbS NPLs. This reflects the heavier mass $m_{x}^{*}$ in orthorhombic band edges (Table 3) as compared to the cubic counterparts (see SI), especially for the valence band.

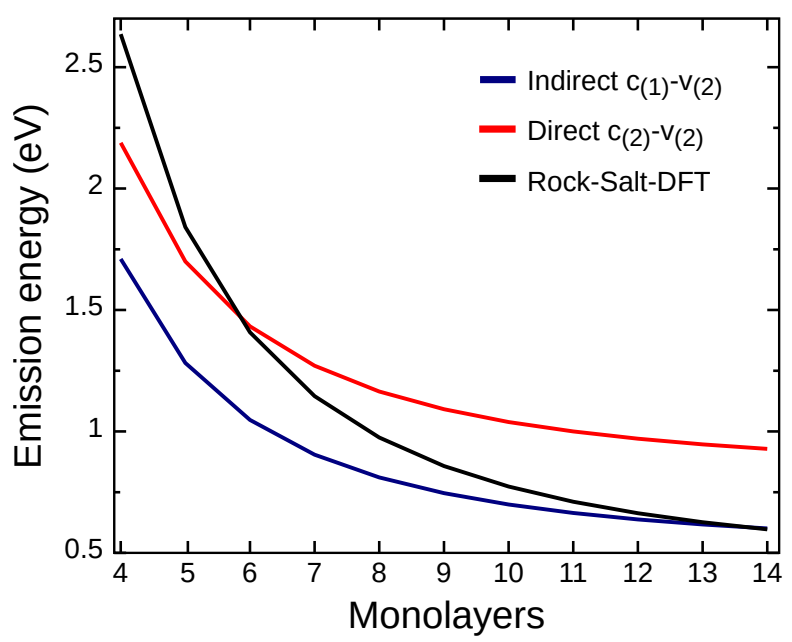

Figure 8: Exciton emission energies vs NPL thickness ( $x$ direction) for direct (red) and indirect (blue) band edge of orthorhombic PbS NPLs, and for rock salt PbS NPLs (black). Lateral dimensions $\left(L_{y}\right.$ and $L_{z}$ ) remain constant at $50 \mathrm{~nm}$.

\section{Discussion}

From the point of view of the electronic structure, the main effect of the orthorhombic distortion of the $\mathrm{PbS}$ lattice is the formation of an indirect gap, between $c_{(1)}$ and $v_{(2)}$ bands. This is a drastic change as compared to rock-salt, where PBEsol predicts a direct band gap instead (see Fig. S3). From Table 2, the energy splitting between the fundamental (indirect) band gap and the direct ones exceeds $0.20 \mathrm{eV}$ in bulk. For NPLs confined along the $a$ crystallographic direction, ${ }^{15,16}$ the masses reported in Table 3 indicate that the gap will open further (e.g. $0.4 \mathrm{eV}$ in Fig. 6a between $c_{(1)^{-}} v_{(2)}$ and $c_{(2)}{ }^{-} v_{(2)}$ ). These splittings are well beyond thermal energy at room temperature. Therefore, in equilibrium most photoexcited electron-hole pairs would occupy states of the indirect gap.

One can try to draw connections between this finding and experimental observations. Orthorhombic NPLs show weak luminescence and high energy absorption onset / emission 
peak $(\sim 1.6 \mathrm{eV}) .{ }^{15,16}$ Yet, time-resolved photoluminescence shows radiative recombination is fast. ${ }^{15}$ The weak luminescence may result from the indirect nature of the fundamental transition. After non-resonant excitations, only a small fraction of photoexcited electron-hole pairs recombines radiatively across direct gaps, while the majority decays to the indirect one. In the direct gap recombination, giant oscillator strength permits short radiative lifetimes. This process should be responsible for the high energy emission and absorption. By contrast, in the indirect gap recombination, optical selection rules yield long radiative lifetime, and subsequent competition with non-radiative relaxation mechanisms leads to low quantum yields.

For 7 monolayer PbS NPLs with weak lateral confinement, we estimate the direct transition energy to be $\sim 1.4 \mathrm{eV}$ (Fig. 6a, red line). This value is slightly lower than the 1.6 $\mathrm{eV}$ in experiments, possibly because DFT understimates actual band gaps, or because the effective NPL thickness is less than the nominal 7 monolayers For comparison, we expect rock salt NPLs with identical thickness to show direct exciton emission at $1.11 \mathrm{eV}$ (Fig. 6a, black line). This would offer a qualitative explanation for the higher radiative energies of orthorhombic NPLs ${ }^{15,16}$ when compared to cubic ones. ${ }^{26,63-65}$ However, the energy difference we estimate is $\Delta E_{g} \sim 0.25 \mathrm{eV}$, well below the $\Delta E_{g}=0.8 \mathrm{eV}$ reported in experiments. It seems likely other factors, such as surface defects or residual nanocrystals, are influencing the different optical response of these objects. The results in a parallel study showing emission near $1.6 \mathrm{eV}$ for cubic PbS NPLs with synthetic refinements reinforce this hypothesis. ${ }^{66}$

Inaccuracy in the determination of the effective NPL thickness may be an issue as well. In both cubic ${ }^{27}$ and orthorhombic (Fig. 6b) NPLs, k·p theory predicts strong excitonic effects. The short radiative lifetimes in emission experiments are consistent with this feature, ${ }^{15,66}$ but in the two systems measured absorption spectra show smeared peaks (see e.g. Fig.S5a). It has been pointed out that uneven NPL thickness, leading to inhomogeneous broadening, may be responsible for this. ${ }^{65}$ If this was the case, the different number of monolayers in the optically active region of the NPL would certainly have a drastic effect on the emission 
wavelength, because of the extreme quantum confinement. This can be observed in Fig. 8 . For nominal thicknesses of 7 monolayers or less, a fluctuation of a single monolayer implies a band edge energy shift exceeding $0.1 \mathrm{eV}$, i.e. comparable to exciton binding energies.

As for the effect of lateral confinement, in Ref. 15 a blueshift of $\sim 30 \mathrm{meV}$ was observed when $L_{z}$ decreased from 12.4 to $3.5 \mathrm{~nm}$, which is well below the $200 \mathrm{meV}$ obtained in our calculations (Fig. 6a). This significant deviation is yet another indication that extrinsic factors, such as impurities or surface roughness, are conditioning the actual photo-physical response. Synthetic efforts to control the NPL defects and homogeneity are then expected to be key in improving the optical properties of orthorhombic PbS NPLs and reach the maturity of their cadmium chalcogenide counterparts. Yet, even if this issues are circumvented, the indirect band gap we predict is an intrinsic factor which will ultimately determine the performance of these structures.

\section{Conclusions}

We have synthesized orthorhombic PbS NPLs and determined the lattice parameters using SAED. We have calculated the band structure of orthorhombic PbS bulk with such parameters, using DFT with PBEsol/PBE functionals and the GW approximation, all with the full SOC inclusion. The electronic structure presents an indirect bandgap, which is reinforced by vertical confinement (along the $a$ axis) in the case of NPLs, as shown by $\mathrm{k} \cdot \mathrm{p}$ calculations. This result may explain the weak luminescence reported by recent experiments with such structures. We derived effective masses for conduction and valence bands, and predict that strong confinement along $b$ or $c$ crystallographic axes could restore the direct band gap nature, possibly improving the luminescence. Calculations including electron-hole correlation reveal strong excitonic effects with binding energies exceeding $100 \mathrm{meV}$ for thin NPLs, driven by quantum and dielectric confinement. These results could have important practical consequences in the development of optoelectronic devices based in PbS NPLs, -e.g. global prospects to improve the luminescence of these structures for NIR detection. 


\section{Acknowledgement}

Financial support from the European Research Council (ERC) via Consolidator Grant (724424-No-LIMIT), Generalitat Valenciana via Prometeo Grant Q-Devices (Prometeo /2018/098), EU (FEDER) and MINECO under project TEC2017-85912-C2-2 is gratefully acknowledged. J.P. and J.I.C. acknowledge support form MICINN project CTQ2017-83781-P. We acknowledge the SCSIE from the University of Valencia for providing HRTEM and SAEDs facilities.

\section{Supporting Information Available}

Effective masses of rock-salt $\mathrm{PbS}$ obtained from $\mathrm{k} \cdot \mathrm{p}$ theory; energy bands of orthorhombic and rock-salt PbS by using $\mathrm{PBE}$ and PBEsol exchange-correlation functional, respectively; histograms of the average length and width of PbS NPLs; UV-Vis absorption and PL spectra of PbS NPLS at different times of reaction; energy bands of quasi-tetragonal PbS with the PBEsol functional.

\section{References}

(1) Hyun, B.-R.; Zhong, Y.-W.; Bartnik, A. C.; Sun, L.; Abruña, H. D.; Wise, F. W.; Goodreau, J. D.; Matthews, J. R.; Leslie, T. M.; Borrelli, N. F. Electron Injection from Colloidal PbS Quantum Dots into Titanium Dioxide Nanoparticles. ACS Nano 2008, 2, 2206-2212, PMID: 19206384.

(2) Carey, G. H.; Abdelhady, A. L.; Ning, Z.; Thon, S. M.; Bakr, O. M.; Sargent, E. H. Colloidal Quantum Dot Solar Cells. Chemical Reviews 2015, 115, 12732-12763, PMID: 26106908.

(3) Sanchez, R. S.; Binetti, E.; Torre, J. A.; Garcia-Belmonte, G.; Striccoli, M.; MoraSero, I. All solution processed low turn-on voltage near infrared LEDs based on core- 
shell PbS-CdS quantum dots with inverted device structure. Nanoscale 2014, 6, 85518555.

(4) De Iacovo, A.; Venettacci, C.; Colace, L.; Scopa, L.; Foglia, S. PbS Colloidal Quantum Dot Photodetectors operating in the near infrared. Scientific Reports 2016, 6, 37913.

(5) Konstantatos, G.; Sargent, E. H. PbS colloidal quantum dot photoconductive photodetectors: Transport, traps, and gain. Applied Physics Letters 2007, 91, 173505.

(6) Ngo, T. T.; Masi, S.; Mendez, P. F.; Kazes, M.; Oron, D.; Seró, I. M. PbS quantum dots as additives in methylammonium halide perovskite solar cells: the effect of quantum dot capping. Nanoscale Advances 2019, 1, 4109-4118.

(7) Han, J.; Luo, S.; Yin, X.; Zhou, Y.; Nan, H.; Li, J.; Li, X.; Oron, D.; Shen, H.; Lin, H. Hybrid PbS Quantum-Dot-in-Perovskite for High-Efficiency Perovskite Solar Cell. Small 2018, 14, 1801016.

(8) Masi, S.; Echeverría-Arrondo, C.; Salim, K. M.; Ngo, T. T.; Mendez, P. F.; LópezFraguas, E.; Macias-Pinilla, D. F.; Planelles, J.; Climente, J. I.; Mora-Sero, I. ChemiStructural Stabilization of Formamidinium Lead Iodide Perovskite by Using Embedded Quantum Dots. ACS Energy Letters 2020, 5, 418-427.

(9) Gong, X.; Yang, Z.; Walters, G.; Comin, R.; Ning, Z.; Beauregard, E.; Adinolfi, V.; Voznyy, O.; Sargent, E. H. Highly efficient quantum dot near-infrared light-emitting diodes. Nature Photonics 2016, 10, 253-257.

(10) García de Arquer, F. P.; Gong, X.; Sabatini, R. P.; Liu, M.; Kim, G.-H.; Sutherland, B. R.; Voznyy, O.; Xu, J.; Pang, Y.; Hoogland, S.; Sinton, D.; Sargent, E. Field-emission from quantum-dot-in-perovskite solids. Nature Communications 2017, 8,14757 . 
(11) YousefiAmin, A.; Killilea, N. A.; Sytnyk, M.; Maisch, P.; Tam, K. C.; Egelhaaf, H.-J.; Langner, S.; Stubhan, T.; Brabec, C. J.; Rejek, T.; Halik, M.; Poulsen, K.; Niehaus, J.; Köck, A.; Heiss, W. Fully Printed Infrared Photodetectors from PbS Nanocrystals with Perovskite Ligands. ACS Nano 2019, 13, 2389-2397, PMID: 30706709.

(12) Lhuillier, E.; Pedetti, S.; Ithurria, S.; Nadal, B.; Heuclin, H.; Dubertret, B. Twodimensional colloidal metal chalcogenides semiconductors: synthesis, spectroscopy, and applications. Accounts of chemical research 2015, 48, 22-30.

(13) Yu, J.; Chen, R. Optical properties and applications of two-dimensional CdSe nanoplatelets. InfoMat 2020, 2, 905-927.

(14) Diroll, B. T. Colloidal quantum wells for optoelectronic devices. Journal of Materials Chemistry C 2020, 8, 10628-10640.

(15) Khan, A. H.; Brescia, R.; Polovitsyn, A.; Angeloni, I.; Martín-García, B.; Moreels, I. Near-Infrared Emitting Colloidal PbS Nanoplatelets: Lateral Size Control and Optical Spectroscopy. Chemistry of Materials 2017, 29, 2883-2889.

(16) Akkerman, Q. A.; Martín-García, B.; Buha, J.; Almeida, G.; Toso, S.; Marras, S.; Bonaccorso, F.; Petralanda, U.; Infante, I.; Manna, L. Ultrathin Orthorhombic PbS Nanosheets. Chemistry of Materials 2019, 31, 8145-8153.

(17) Schliehe, C.; Juarez, B. H.; Pelletier, M.; Jander, S.; Greshnykh, D.; Nagel, M.; Meyer, A.; Foerster, S.; Kornowski, A.; Klinke, C.; Weller, H. Ultrathin PbS sheets by two-dimensional oriented attachment. Science 2010, 329, 550-553.

(18) Aerts, M.; Bielewicz, T.; Klinke, C.; Grozema, F. C.; Houtepen, A. J.; Schins, J. M.; Siebbeles, L. D. A. Highly efficient carrier multiplication in PbS nanosheets. Nature Communications 2014, 5, 3789. 
(19) Ramin Moayed, M. M.; Bielewicz, T.; Zöllner, M. S.; Herrmann, C.; Klinke, C. Towards colloidal spintronics through Rashba spin-orbit interaction in lead sulphide nanosheets. Nature Communications 2017, 8, 15721.

(20) Morrison, P. J.; Loomis, R. A.; Buhro, W. E. Synthesis and Growth Mechanism of Lead Sulfide Quantum Platelets in Lamellar Mesophase Templates. Chemistry of Materials 2014, 26, 5012-5019.

(21) Movilla, J. L.; Planelles, J.; Climente, J. I. Dielectric Confinement Enables Molecular Coupling in Stacked Colloidal Nanoplatelets. The Journal of Physical Chemistry Letters 2020, 11, 3294-3300.

(22) Khan, A. H.; Pal, S.; Dalui, A.; Pradhan, J.; Sarma, D.; Acharya, S. Solution-processed free-standing ultrathin two-dimensional $\mathrm{PbS}$ nanocrystals with efficient and highly stable dielectric properties. Chemistry of Materials 2017, 29, 1175-1182.

(23) Li, H.; Zhitomirsky, D.; Grossman, J. C. Tunable and energetically robust PbS nanoplatelets for optoelectronic applications. Chemistry of Materials 2016, 28, 18881896.

(24) Wan, W.; Yao, Y.; Sun, L.; Liu, C.-C.; Zhang, F. Topological, valleytronic, and optical properties of monolayer PbS. Advanced Materials 2017, 29, 1604788.

(25) Sánchez-Godoy, H. E.; Erazo, E. A.; Gualdrón-Reyes, A. F.; Khan, A. H.; Agouram, S.; Barea, E. M.; Rodriguez, R. A.; Zarazúa, I.; Ortiz, P.; Cortés, M. T.; MuñozSanjosé, V.; Moreels, I.; Masi, S.; Mora-Seró, I. Preferred Growth Direction by PbS Nanoplatelets Preserves Perovskite Infrared Light Harvesting for Stable, Reproducible, and Efficient Solar Cells. Advanced Energy Materials 2020, 10, 2002422.

(26) Bhandari, G. B.; Subedi, K.; He, Y.; Jiang, Z.; Leopold, M.; Reilly, N.; Lu, H. P.; Zayak, A. T.; Sun, L. Thickness-controlled synthesis of colloidal PbS nanosheets and their thickness-dependent energy gaps. Chemistry of Materials 2014, 26, 5433-5436. 
(27) Yang, J.; Wise, F. Electronic states of lead-salt nanosheets. The Journal of Physical Chemistry C 2015, 119, 26809-26816.

(28) Deb, A. K.; Kumar, V. Bandgap engineering in semiconducting one to few layers of SnS and SnSe. Physica status solidi (b) 2017, 254, 1600379.

(29) Zagorac, D.; Doll, K.; Schön, J.; Jansen, M. Ab initio structure prediction for lead sulfide at standard and elevated pressures. Physical Review B 2011, 84, 045206.

(30) Ithurria, S.; Tessier, M.; Mahler, B.; Lobo, R.; Dubertret, B.; Efros, A. L. Colloidal nanoplatelets with two-dimensional electronic structure. Nature materials 2011, 10, 936-941.

(31) Christodoulou, S.; Climente, J. I.; Planelles, J.; Brescia, R.; Prato, M.; MartínGarcía, B.; Khan, A. H.; Moreels, I. Chloride-Induced Thickness Control in CdSe Nanoplatelets. Nano Letters 2018, 18, 6248-6254.

(32) Yakovkin, I. N.; Dowben, P. A. The problem of the band gap in LDA calculations. Surface Review and Letters 2007, 14, 481-487.

(33) Grzechnik, A.; Friese, K. Pressure-induced orthorhombic structure of PbS. Journal of Physics: Condensed Matter 2010, 22, 095402.

(34) Perdew, J. P.; Ruzsinszky, A.; Csonka, G. I.; Vydrov, O. A.; Scuseria, G. E.; Constantin, L. A.; Zhou, X.; Burke, K. Restoring the density-gradient expansion for exchange in solids and surfaces. Physical review letters 2008, 100, 136406.

(35) Perdew, J. P.; Burke, K.; Ernzerhof, M. Generalized gradient approximation made simple. Physical review letters 1996, 77, 3865.

(36) Bose, S.; Song, Z.; Fan, W. J.; Zhang, D. H. Effect of lateral size and thickness on the electronic structure and optical properties of quasi two-dimensional CdSe and CdS nanoplatelets. Journal of Applied Physics 2016, 119, 143107. 
(37) Giannozzi, P. et al. QUANTUM ESPRESSO: a modular and open-source software project for quantum simulations of materials. Journal of Physics: Condensed Matter 2009, 21, 395502.

(38) Wentzcovitch, R. M. Invariant molecular-dynamics approach to structural phase transitions. Physical Review B 1991, 44, 2358.

(39) Marini, A.; Hogan, C.; Grüning, M.; Varsano, D. Yambo: an ab initio tool for excited state calculations. Computer Physics Communications 2009, 180, 1392-1403.

(40) Sangalli, D. et al. Many-body perturbation theory calculations using the yambo code. Journal of Physics: Condensed Matter 2019, 31, 325902.

(41) Hedin, L. New method for calculating the one-particle Green's function with application to the electron-gas problem. Physical Review 1965, 139, A796.

(42) Aryasetiawan, F.; Gunnarsson, O. The GW method. Reports on Progress in Physics $1998,61,237$.

(43) Rajadell, F.; Climente, J. I.; Planelles, J. Excitons in core-only, core-shell and corecrown CdSe nanoplatelets: Interplay between in-plane electron-hole correlation, spatial confinement, and dielectric confinement. Physical Review B 2017, 96, 035307.

(44) Benchamekh, R.; Gippius, N. A.; Even, J.; Nestoklon, M.; Jancu, J.-M.; Ithurria, S.; Dubertret, B.; Efros, A. L.; Voisin, P. Tight-binding calculations of image-charge effects in colloidal nanoscale platelets of CdSe. Physical Review B 2014, 89, 035307.

(45) Achtstein, A. W.; Schliwa, A.; Prudnikau, A.; Hardzei, M.; Artemyev, M. V.; Thomsen, C.; Woggon, U. Electronic Structure and Exciton-Phonon Interaction in TwoDimensional Colloidal CdSe Nanosheets. Nano Letters 2012, 12, 3151-3157.

(46) Planelles, J. Simple correlated wave-function for excitons in 0D, quasi-1D and quasi-2D quantum dots. Theoretical Chemistry Accounts 2017, 136, 81. 
(47) Morrison, P. J.; Loomis, R. A.; Buhro, W. E. Synthesis and growth mechanism of lead sulfide quantum platelets in lamellar mesophase templates. Chemistry of Materials 2014, 26, 5012-5019.

(48) Momma, K.; Izumi, F. VESTA 3 for three-dimensional visualization of crystal, volumetric and morphology data. Journal of applied crystallography 2011, 44, 1272-1276.

(49) Li, F.; Liu, X.; Wang, Y.; Li, Y. Germanium monosulfide monolayer: a novel twodimensional semiconductor with a high carrier mobility. Journal of Materials Chemistry C 2016, 4, 2155-2159.

(50) Li, Z.; Chen, G.; Liu, J. Electron structure and optical absorption properties of cubic and orthorhombic NaTaO3 by density functional theory. Solid state communications 2007, 143, 295-299.

(51) Nakamura, T.; Suemasu, T.; Takakura, K.-i.; Hasegawa, F.; Wakahara, A.; Imai, M. Investigation of the energy band structure of orthorhombic BaSi 2 by optical and electrical measurements and theoretical calculations. Applied physics letters 2002, 81, 1032-1034.

(52) Hummer, K.; Grüneis, A.; Kresse, G. Structural and electronic properties of lead chalcogenides from first principles. Physical Review B 2007, 75, 195211.

(53) Allan, G.; Delerue, C. Confinement effects in PbSe quantum wells and nanocrystals. Physical Review B 2004, 70, 245321.

(54) Peterson, J. J.; Huang, L.; Delerue, C.; Allan, G.; Krauss, T. D. Uncovering forbidden optical transitions in PbSe nanocrystals. Nano Letters 2007, 7, 3827-3831.

(55) Kittel, C. Introduction to solid state physics, 8th ed.; John Wiley \& Sons: New York, NY, 2004; Chapter 8, pp 197-217.

(56) Green, M. A. Intrinsic concentration, effective densities of states, and effective mass in silicon. Journal of Applied Physics 1990, 67, 2944-2954. 
(57) Nanda, K.; Kruis, F.; Fissan, H.; Behera, S. Effective mass approximation for two extreme semiconductors: Band gap of $\mathrm{PbS}$ and $\mathrm{CuBr}$ nanoparticles. Journal of applied physics 2004, 95, 5035-5041.

(58) Cardona, M.; Peter, Y. Y. Fundamentals of Semiconductors, 3rd ed.; Springer-Verlag Berlin Heidelberg, 1996; Chapter 2, pp 63-89.

(59) Kang, I.; Wise, F. W. Electronic structure and optical properties of PbS and PbSe quantum dots. JOSA B 1997, 14, 1632-1646.

(60) Gupta, B.; Kumar, V. Analysis of effective compressibilities in PbS, PbSe, PbTe and SnTe. Solid State Communications 1983, 45, 745-747.

(61) Lüdde, K. Das dielektrische und refraktodensimetrische Verhalten flüssiger Fette beim Altern. Fette, Seifen, Anstrichmittel 1959, 61, 1156-1163.

(62) Achtstein, A. W.; Schliwa, A.; Prudnikau, A.; Hardzei, M.; Artemyev, M. V.; Thomsen, C.; Woggon, U. Electronic structure and exciton-phonon interaction in twodimensional colloidal CdSe nanosheets. Nano letters 2012, 12, 3151-3157.

(63) Zhang, H.; Savitzky, B. H.; Yang, J.; Newman, J. T.; Perez, K. A.; Hyun, B.-R.; Kourkoutis, L. F.; Hanrath, T.; Wise, F. W. Colloidal synthesis of PbS and PbS/CdS nanosheets using acetate-free precursors. Chemistry of Materials 2016, 28, 127-134.

(64) Jiang, Z.; Bhandari, G. B.; Premathilaka, S. M.; Khan, S.; Dimick, D. M.; Stombaugh, C.; Mandell, A.; He, Y.; Lu, H. P.; Sun, L. Growth of colloidal PbS nanosheets and the enhancement of their photoluminescence. Physical Chemistry Chemical Physics 2015, 17, 23303-23307.

(65) Antu, A. D.; Jiang, Z.; Premathilka, S. M.; Tang, Y.; Hu, J.; Roy, A.; Sun, L. Bright colloidal PbS nanoribbons. Chemistry of Materials 2018, 30, 3697-3703. 
(66) Vázquez, F. M.; Yu, Q.; Klepzig, L. F.; Siebbeles, L. D.; Crisp, R. W.; Lauth, J. Probing Excitons in Ultrathin PbS Nanoplatelets with Enhanced Near-Infrared Emission. Submitted on 14 Nov 2020. arxiv (physics.optics) 2011.07174v2, (Accessed 2020-12-09). 
Graphical TOC Entry

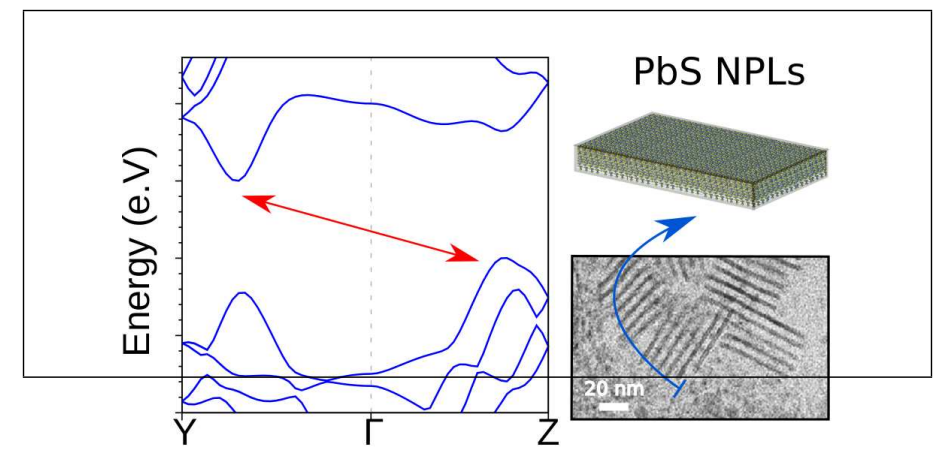




\section{Supplementary Information}

\section{Morphology and band structure of orthorhombic PbS nanoplatelets: an indirect band gap material}

David F. Macias-Pinilla, ${ }^{\dagger \neq}$ Carlos Echeverría-Arrondo, ${ }^{\ddagger}$ Andrés Fabián Gualdrón

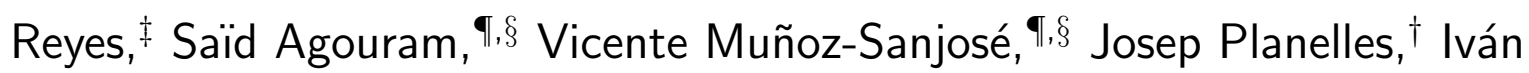
Mora-Seró, $\neq, \delta$ and Juan I. Climente ${ }^{*, \dagger}$

$\dagger$ Departament de Química Física i Analítica, Universitat Jaume I, Av. Sos Baynat, s/n, 12071 Castelló, Spain

$\ddagger$ Institute of Advanced Materials (INAM), Universitat Jaume I, Av. Sos Baynat, s/n, 12071 Castelló, Spain

IDepartment of Applied Physics and Electromagnetism, University of Valencia, 46100 Valencia, Spain §Materials for Renewable Energy (MAER), Unitat Mixa d'Investigació UV-UJI

E-mail: climente@uji.es

\section{PbS Rock-Salt Effective Masses via k · p Model}

In order to understand the photophysics of the PbS rock-salt NPLs we take advantage of the Dimmock Hamiltonian, which is the expansion of the $k \cdot p$ Hamiltonian, including valenceconduction interaction, anisotropies, self polarization potential and SOC. ${ }^{1}$ A full view of the model used in $\mathrm{PbS}$ rock-salt structures can be found in the supplementary information of Ref. 2. Rock-salt PbS has direct bandgap at $L$ point (center of the hexagonal face of 
the first Brillouin zone). With $z$ in the cubic lattice direction [111], the valence band (VB) edge Bloch function has $L_{6}^{+}$symmetry ( $s$-like) and the conduction band (CB) edge Bloch function has $L_{6}^{-}$symmetry $\left(p_{z}\right.$-like). With theses considerations we can write the Dimmock Hamiltonian as a $4 \times 4$ matrix (conduction and valence - spin up, spin down) ${ }^{1}$

$$
H=\left[\begin{array}{cccc}
\frac{E_{g}}{2}+\frac{\hbar^{2} k_{t}^{2}}{2 m_{t}^{-}}+\frac{\hbar^{2} k_{z}^{2}}{2 m_{l}^{-}} & 0 & \frac{\hbar}{m} P_{l} k_{z} & \frac{\hbar}{m} P_{t}\left(k_{x}-i k_{y}\right) \\
0 & \frac{E_{g}}{2}+\frac{\hbar^{2} k_{t}^{2}}{2 m_{t}^{-}}+\frac{\hbar^{2} k_{z}^{2}}{2 m_{l}^{-}} & \frac{\hbar}{m} P_{t}\left(k_{x}+i k_{y}\right) & -\frac{\hbar}{m} P_{l} k_{z} \\
\frac{\hbar}{m} P_{l} k_{z} & \frac{\hbar}{m} P_{t}\left(k_{x}-i k_{y}\right) & -\frac{E_{g}}{2}-\frac{\hbar^{2} k_{t}^{2}}{2 m_{t}^{+}}-\frac{\hbar^{2} k_{z}^{2}}{2 m_{l}^{+}} & 0 \\
\frac{\hbar}{m} P_{t}\left(k_{x}+i k_{y}\right) & -\frac{\hbar}{m} P_{l} k_{z} & 0 & -\frac{E_{g}}{2}-\frac{\hbar^{2} k_{t}^{2}}{2 m_{t}^{+}}-\frac{\hbar^{2} k_{z}^{2}}{2 m_{l}^{+}}
\end{array}\right],
$$

where $E_{g}$ is the band gap, $m$ is the free electron mass, $P_{t}$ and $P_{l}$ are the transverse and longitudinal Kane parameters, $m_{t}^{ \pm}$and $m_{l}^{ \pm}$are the transverse and longitudinal band-edge effective masses for electron $(-)$ and hole $(+)$, and $k_{i}$ are momentum operators.

Hamiltonian in eq. (1) can be written in compact form as in Ref. 3

$$
H=\left[\begin{array}{cc}
\left(\frac{E_{g}}{2}+\frac{\hbar^{2} k_{t}^{2}}{2 m_{t}^{-}}+\frac{\hbar^{2} k_{z}^{2}}{2 m_{l}^{-}}\right) \mathbb{1} & \frac{\hbar}{m} P_{l} k_{z} \sigma_{z}+\frac{\hbar}{m} P_{t} k_{t} \sigma_{t} \\
\frac{\hbar}{m} P_{l} k_{z} \sigma_{z}+\frac{\hbar}{m} P_{t} k_{t} \sigma_{t} & \left(-\frac{E_{g}}{2}-\frac{\hbar^{2} k_{t}^{2}}{2 m_{t}^{+}}-\frac{\hbar^{2} k_{z}^{2}}{2 m_{l}^{+}}\right) \mathbb{1}
\end{array}\right],
$$

where $\sigma_{x}, \sigma_{y}$ and $\sigma_{z}$ are the Pauli matrices, $k_{t}=\left(k_{x}, k_{y}\right), \sigma_{t}=\left(\sigma_{x}, \sigma_{y}\right)$, and $\mathbb{1}$ is the $2 \times 2$ unit matrix. We can abbreviate the notation and write

$$
H=\left[\begin{array}{cc}
h_{c} & h_{c v} \\
h_{v c} & h_{v}
\end{array}\right]
$$

with $h_{c v}=h_{v c}$.

Our goal is to obtain effective, single-band Hamiltonians for conduction and valence bands, and the associated effective masses, from the multi-band Dimmock Hamiltonian, eq. 
(1). The detail procedure is explained below. Similar reasoning have been used in Ref. 3 . The eigenvalue equation from eq. (2) reads

$$
\left[\begin{array}{cc}
h_{c} & h_{c v} \\
h_{v c} & h_{v}
\end{array}\right]\left(\begin{array}{c}
F_{c} \\
F_{v}
\end{array}\right)=E\left(\begin{array}{c}
F_{c} \\
F_{v}
\end{array}\right),
$$

where $F c$ and $F v$ are the envelope function of conduction and valence band, respectively. Equation (3) is equivalent to equation set

$$
\begin{aligned}
& h_{c} F_{c}+h_{c v} F_{v}=E F_{c}, \\
& h_{v c} F_{c}+h_{v} F_{v}=E F_{v} .
\end{aligned}
$$

From equation (5), we have $\left(E-h_{v}\right) F_{v}=h_{v c} F_{c} \rightarrow F_{v}=\left(E-h_{v}\right)^{-1} h_{v c} F_{c}$, replacing the last term in equation (4) we obtain an effective Hamiltonian for the conduction band

$$
\left[h_{c}+h_{c v}\left(E-h_{v}\right)^{-1} h_{v c}\right] F_{c}=E F_{c}
$$

Similarly, for the valence band one obtains

$$
\left[h_{v}+h_{v c}\left(E-h_{c}\right)^{-1} h_{c v}\right] F_{v}=E F_{v} .
$$

If we write $\Delta=\left(\frac{E_{g}}{2}+\frac{\hbar^{2} k_{t}^{2}}{2 m_{t}^{+}}+\frac{\hbar^{2} k_{z}^{2}}{2 m_{l}^{+}}\right)$, then $h_{v}=-\Delta \mathbb{1}$ and $\left(E-h_{v}\right)^{-1}=\frac{1}{E+\Delta} \mathbb{1}$. Carriers in NPLs are confined within it, then if we consider the case of confinement in the $z$ direction, $\left(E-h_{v}\right)^{-1}=F \mathbb{1}$, and $F=\frac{1}{E+\Delta}$.

With these considerations, and simplifying the notation $\frac{\hbar}{m} P \equiv P$, then we can write 


$$
\begin{aligned}
h_{c v}\left(E-h_{v}\right)^{-1} h_{v c} & =\left[P_{l} k_{z} \sigma_{z}+P_{t} k_{x} \sigma_{x}+P_{t} k_{y} \sigma_{y}\right] F \\
& \times\left[P_{l} k_{z} \sigma_{z}+P_{t} k_{x} \sigma_{x}+P_{t} k_{y} \sigma_{y}\right] \\
& =F\left[P_{l}^{2} k_{z}^{2}+P_{t}^{2} k_{t}^{2}\right] \mathbb{1}+P_{l}^{2} k_{z} F k_{z} \mathbb{1} \\
& +i \sigma_{y}\left(F P_{l} P_{t} k_{z} k_{x}-F P_{l} P_{t} k_{x} k_{z}\right) \\
& +i \sigma_{y} P_{l} P_{t} k_{z} F k_{x}+i \sigma_{x}\left(F P_{t} P_{l} k_{y} k_{z}\right. \\
& \left.-F P_{l} P_{t} k_{z} k_{y}\right)-i \sigma_{x} P_{l} P_{t} k_{z} F k_{y} \\
& +i \sigma_{z}\left(F P_{t}^{2} k_{x} k_{y}-F P_{t}^{2} k_{y} k_{x}\right) .
\end{aligned}
$$

Due to commutativity of the linear momentum components, most terms disappear from the above equation, and we have

$$
\begin{aligned}
h_{c v}\left(E-h_{v}\right)^{-1} h_{v c} & =\mathbb{1}\left(P_{l}^{2} k_{z} F k_{z}+P_{t}^{2} F k_{t}^{2}\right) \\
& -i P_{l} P_{t} k_{z}(F)\left(\sigma \times k_{t} \cdot \hat{z}\right),
\end{aligned}
$$

where $\hat{z}$ is a unitary vector on $z$ direction. The second term is of Rashba type ${ }^{4,5}$ and vanishes except in asymmetric heterojunctions. Thus equation (6) is given by

$$
\left[\frac{E_{g}}{2}+\frac{\hbar^{2} k_{t}^{2}}{2 m_{t}^{e}}+\frac{\hbar^{2} k_{z}^{2}}{2 m_{l}^{e}}\right] F_{c}=E F_{c}
$$

where $m_{t}^{e}=\left(\frac{1}{m_{t}^{-}}+\frac{2 P_{t}^{2} F}{m^{2}}\right)$ and $m_{l}^{e}=\left(\frac{1}{m_{l}^{-}}+\frac{2 P_{l}^{2} F}{m^{2}}\right)$. Then the effective Hamiltonian, in the single band approximation, looks like a variable mass Hamiltonian with masses having the energy eigenvalues from the 4-band Dimmock Hamiltonian, which allows us to introduce the interaction between conduction and valence bands (similar results are obtained for the valence band from eq. 7). So, considering that the masses are only variable with the position, we have 


$$
H=-\frac{\hbar^{2}}{2}\left(\frac{d}{d x} \frac{1}{m_{\perp}} \frac{d}{d x}+\frac{d}{d y} \frac{1}{m_{\perp}} \frac{d}{d y}+\frac{d}{d z} \frac{1}{m_{z}} \frac{d}{d z}\right)+V(x, y, z)
$$

The effective masses of a single band can be obtained, but ultimately depend on the energies of the multiband calculation. Knowing that the substitution is possible, for efficiency reasons instead of substituting one by one the terms that relate the single band masses with the terms of the multiband, we will make calculations of a single band and we will find which effective masses fit better to those obtained by the multiband Dimmock calculation.
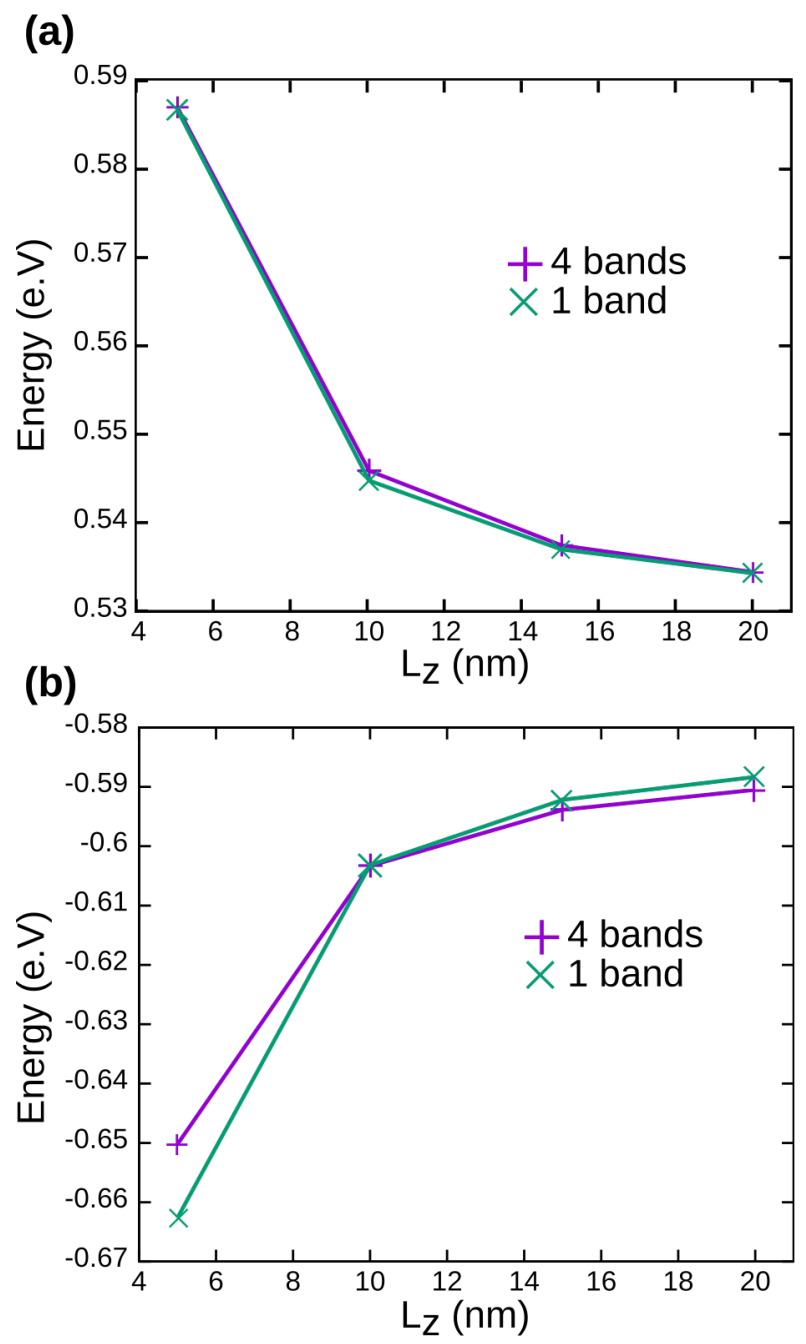

Figure S1: Electron (a) and hole (b) energy as a function of the NPL lateral confinement. The single-band result is the best fit to the full (four-band) calculation, which allows us to propose effective masses. The effective masses found by the single Hamiltonian fit are $m_{x}^{*}=0.29\left(m_{x}^{*}=0.25\right)$ and $m_{y z}^{*}=0.27\left(m_{y z}^{*}=0.19\right)$ for electrons (holes). 
In Fig. S1. we fit the effective masses, from the single band Hamiltonian, that have the smallest deviation compared to the calculation of 4-bands Hamiltonian in the $L_{z}$ range. In this case, the energy was calculated for a NPL with dimensions $L_{x}=1.8 \mathrm{~nm}, L_{y}=50 \mathrm{~nm}$. Parameters used in Dimmock Hamiltonian are reported in Ref. 1. The effective masses found by the single Hamiltonian fit are $m_{x}^{*}=0.29$ and $m_{y z}^{*}=0.27$ for electrons and $m_{x}^{*}=0.25$ and $m_{y z}^{*}=0.19$ for holes. These masses are similar to those obtained for orthorhombic $\mathrm{PbS}$ using DFT calculations (Table III in the main text).

\section{Energy Bands with PBE functional}

Here we show the energy bands (Fig. S2.) and the effective masses obtained by the PBE functional (TABLE S1), which are similar to those obtained by the PBEsol functional (Fig. 3 main text).

\section{(a) PbS bands}

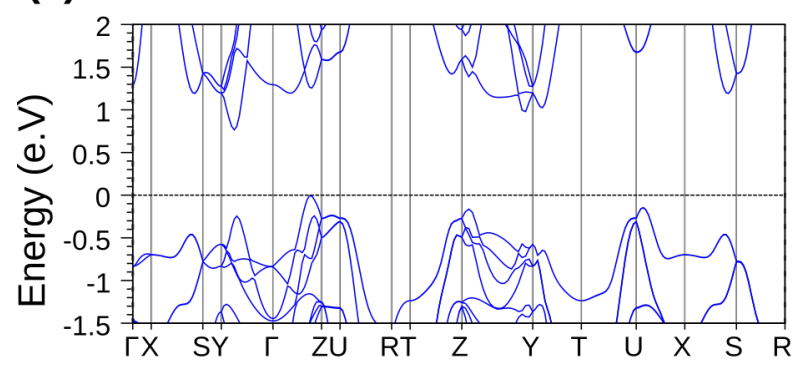

(b) PbS bands $+\mathrm{SOC}$

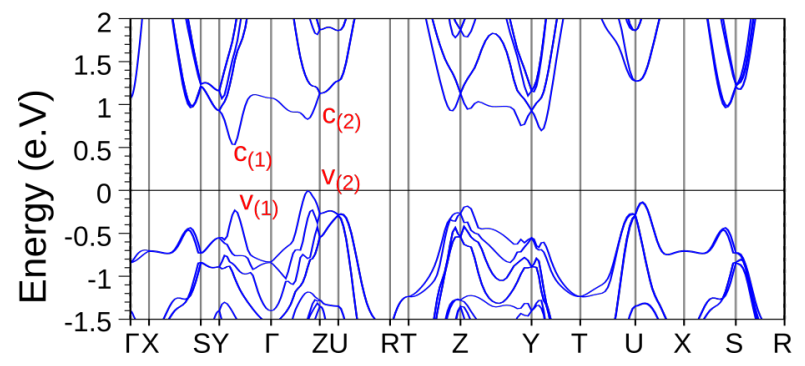

Figure S2: (a) Energy bands without and (b) with SOC by PBE functional. 
Table S1: Effective Masses obtained from energy bands with PBE+SOC. $m_{0}$ is the free electron mass.

\begin{tabular}{cccc}
\multirow{2}{*}{ BAND } & \multicolumn{2}{c}{ Effective Mass } & $\left(\mathbf{m}_{\mathbf{0}}\right)$ \\
& $\mathbf{m}_{\mathbf{x}}^{*}$ & $\mathbf{m}_{\mathbf{y}}^{*}$ & $\mathbf{m}_{\mathbf{z}}^{*}$ \\
\hline $\mathrm{c}_{(1)}$ & 0.303 & 0.149 & 0.159 \\
$\mathrm{c}_{(2)}$ & 0.286 & 0.251 & 0.282 \\
$\mathrm{v}_{(1)}$ & 0.405 & 0.156 & 0.171 \\
$\mathrm{v}_{(2)}$ & 0.70 & 0.248 & 0.206
\end{tabular}

\section{Rock-Salt PbS Band Structure}

We calculate energy bands for the rock-salt structure with the PBEsol functional and the SOC inclusion. We relaxed the structure until forces less than $0.001 R_{y} / a_{0}$. The first Brillouin zone was sampled with a $\Gamma$-centered Monkhorst-pack grid of 6 x6x6 k points. A direct gap $E_{g}=0.24 \mathrm{e} . \mathrm{V}$ is found at the point of symmetry $L$, which is clearly underestimated with respect to the reported experimental value $(0.42 \mathrm{e.V})$.

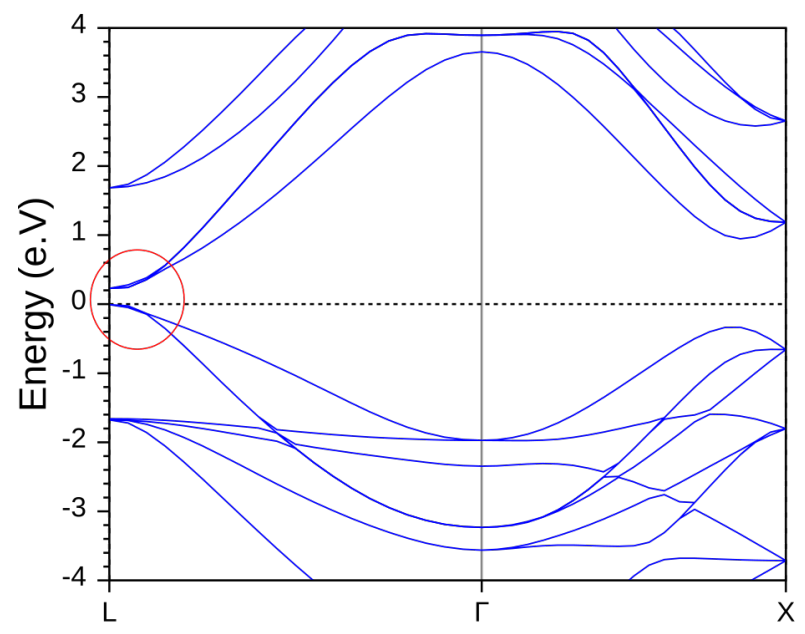

Figure S3: (a) Energy bands for PbS rock-salt structure from the PBEsol functional and SOC.

From energy bands we obtained the effective masses $m_{x}^{*}=0.31\left(m_{x}^{*}=0.25\right)$ and $m_{y z}^{*}=$ $0.27\left(m_{y z}^{*}=0.32\right)$ for electrons (holes). These effective masses are similar to those obtained by fitting to a single band (section I) evidencing the validity of the model and reinforcing the value of the effective masses obtained for the orthorhombic case. 


\section{Experimental Characterization}

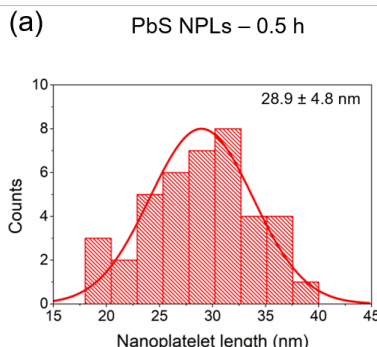

(a')

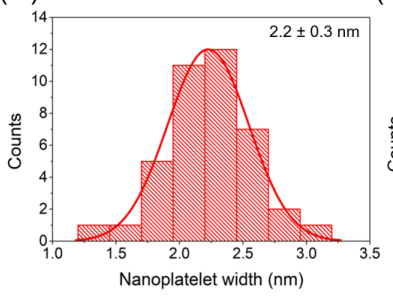

(b) PbS NPLs $-1.0 \mathrm{~h}$

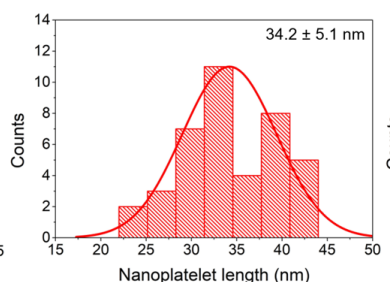

(b')

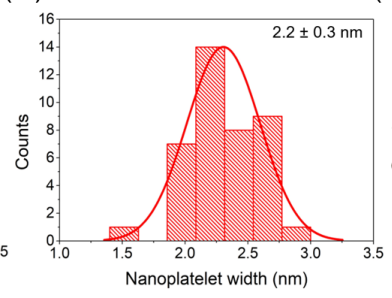

(c) PbS NPLs $-3.0 \mathrm{~h}$

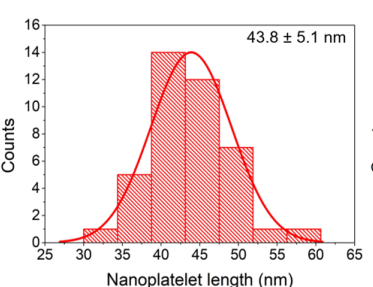

(c')

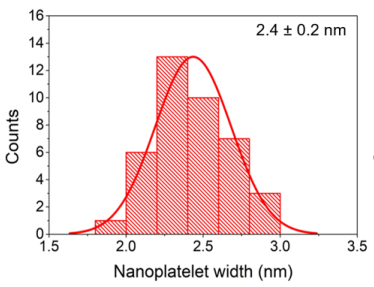

(d) PbS NPLs $-5.0 \mathrm{~h}$

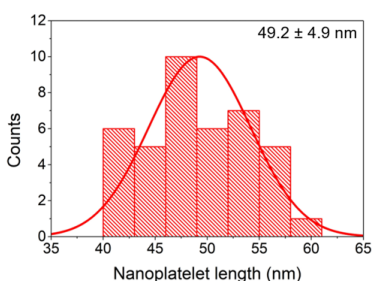

(d')

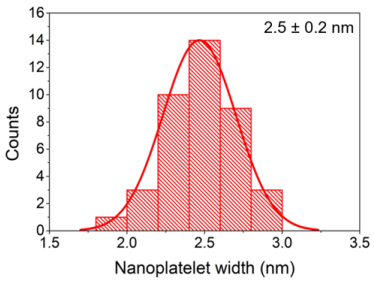

Figure S4: Histograms of the average length and width of PbS NPLs synthesized at different times of reaction: (a,a') $0.5 \mathrm{~h},\left(\mathbf{b}, \mathbf{b}^{\prime}\right) 1.0 \mathrm{~h},\left(\mathbf{c}, \mathbf{c}^{\prime}\right) 3.0 \mathrm{~h}$ and $\left(\mathbf{d}, \mathbf{d}^{\prime}\right) 5.0 \mathrm{~h}$.

(a)

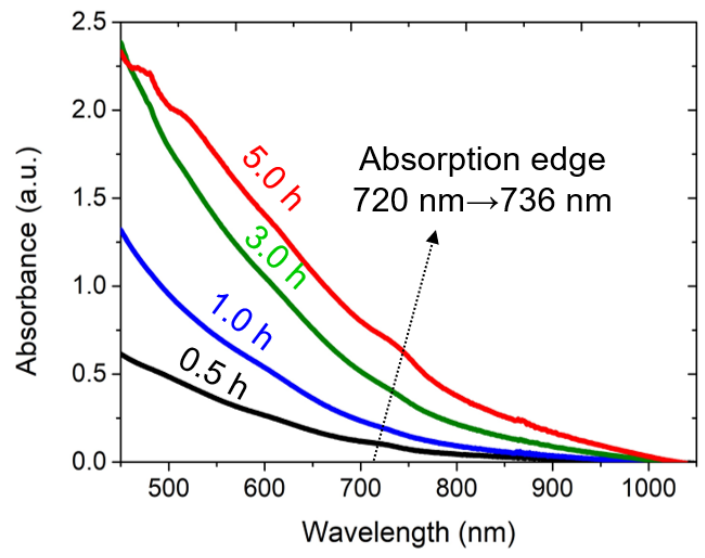

(b)

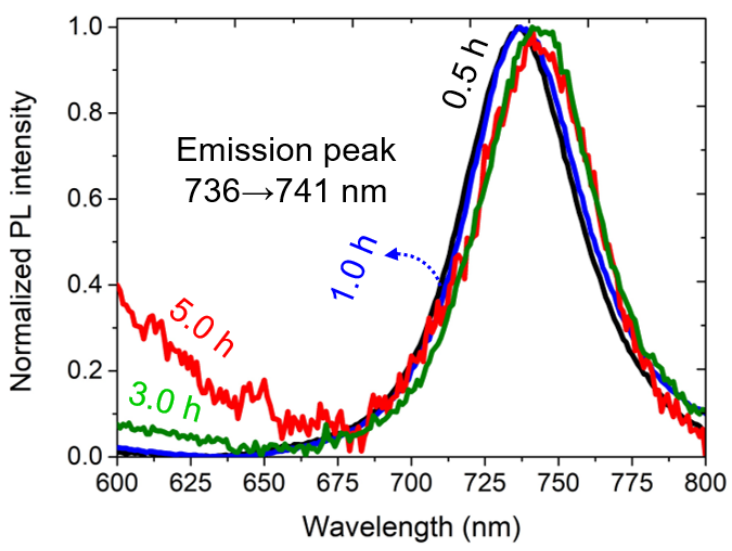

Figure S5: UV-Vis absorption and PL spectra of the PbS NPLs synthesized at different times of reaction: $0.5 \mathrm{~h}, 1.0 \mathrm{~h}, 3.0 \mathrm{~h}$ and $5.0 \mathrm{~h}$. 


\section{Energy Bands of Quasi-tetragonal cell parameters}

We calculate the energy bands of bulk PbS from cell parameters $a=11.9 \AA, b=4.22 \AA$, $c=4.2 \AA,{ }^{6}$ with the PBEsol functional and SOC. In Fig. S6 a direct gap is appreciated and the energy bands are in close agreement with those reported in Ref. 6 by the hybrid HSE06 exchange-correlation functional.

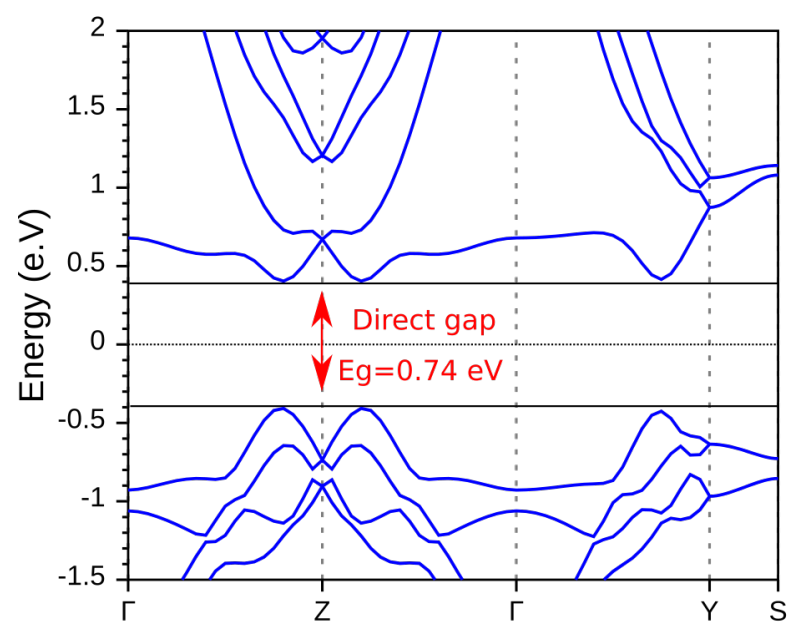

Figure S6: PbS bulk energy bands with quasi-tetragonal cell parameters, PBEsol functional and SOC.

\section{References}

(1) Kang, I.; Wise, F. W. Electronic structure and optical properties of PbS and PbSe quantum dots. JOSA B 1997, 14, 1632-1646.

(2) Masi, S.; Echeverría-Arrondo, C.; Salim, K. M.; Ngo, T. T.; Mendez, P. F.; LópezFraguas, E.; Macias-Pinilla, D. F.; Planelles, J.; Climente, J. I.; Mora-Sero, I. ChemiStructural Stabilization of Formamidinium Lead Iodide Perovskite by Using Embedded Quantum Dots. ACS Energy Letters 2020, 5, 418-427.

(3) Yang, J.; Wise, F. Electronic states of lead-salt nanosheets. The Journal of Physical Chemistry C 2015, 119, 26809-26816. 
(4) Winkler, R. Spin-orbit coupling effects in two-dimensional electron and hole systems. Springer Tracts in Modern Physics 2003, 191, 1-8.

(5) Cardona, M.; Peter, Y. Y. Fundamentals of Semiconductors, 3rd ed.; Springer-Verlag Berlin Heidelberg, 1996; Chapter 2, pp 63-89.

(6) Akkerman, Q. A.; Martín-García, B.; Buha, J.; Almeida, G.; Toso, S.; Marras, S.; Bonaccorso, F.; Petralanda, U.; Infante, I.; Manna, L. Ultrathin Orthorhombic PbS Nanosheets. Chemistry of Materials 2019, 31, 8145-8153. 